\title{
Immunoinducible carbon dots-incorporated hydrogel as photothermal-derived antigen depot to trigger robust antitumor immune response
}

\section{Suwan Liu}

The Affiliated People's Hospital, Jiangsu University

Jingjin Liu

Jiangsu University

Yunchao Wu

The Third People's Hospital of Changzhou

Haoran Wang

Jiangsu University

Xiao Xu

Jiangsu University

Qianzhe Li

Jiangsu University

Mengyu Yang

Jiangsu University

Aihua Gong

Jiangsu University

Miaomiao Zhang

Jiangsu University

Fengyi Du ( $\sim$ biodfy@qq.com)

Jiangsu University

\section{Research Article}

Keywords:

Posted Date: March 8th, 2022

DOI: https://doi.org/10.21203/rs.3.rs-1383156/v1

License: (c) (1) This work is licensed under a Creative Commons Attribution 4.0 International License.

Read Full License 


\section{Abstract}

Photothermal therapy (PTT) causes immunogenic tumor cell death (ICD), while fails to elicit a strong antitumor immune response partially due to its inherent immunosuppressive microenvironment and low antigen presentation. To address these issues, we developed an immunoinducible carbon dotsincorporated hydrogels (iCD@Gel) through a dynamic covalent Schiff base reaction using mannose modifed aluminum-doped carbon dots (M/A-CDs) as cross-linking agent. The M/A-CDs not only possessed superior photothermal conversion efficiency, but also served as nanocarrier to load CpG ODNs for inducing the maturation of dendritic cells (DCs) via manose receptor-mediated targeting delivery. Upon intratumoral injection, the as-prepared iCD@Gel induced immunogentic tumor cell death and damage-related molecular patterns (DAMPs) release via photothermal ablation under $808 \mathrm{~nm}$ NIR irradiation. Subsequently, the iCD@Gel synergized with the DAMPs to significantly promote the maturation and antigen cross-presentation of DCs. Taken together, this work provides a promising strategy to develop nanoparticle-based therapetuic hydrogel for tumor immunotherapy.

\section{Introduction}

Photothermal therapy (PTT) synergized with immunotherapy has revolutionized the cancer treatment [1, 2]. In the process of tumor treatment, PTT not only leads to thermal ablation of tumor cells, but also triggers the anti-tumor immune response via the release of immunogenic cell death (ICB)-mediated damage-related molecular patterns (DAMPs) $[3,4]$. These DAMPs facilitate the recruitment and activation of antigen presenting cells (APCs) to activate strongly $T$ cell-mediated anti-tumor immune responses $[5,6]$. However, PTT-triggered ICD cannot inspire an intense immune response because of the intrinsic immnosuppressive microenvironment and low antigen presentation [7]. The introduction of immune adjuvants in cancer therapy is considered to an effective strategy to amplify the PTT-mediated anti-tumor immune response $[8,9]$.

It has demonstrated that immune adjuvants synergized with PTT could activate the APCs to trigger intense congenital and adaptive immune response [10]. Cytosine-phosphate-guanine oligodeoxynucleotide (CpG-ODN) is a TLR9 receptor agonist which located on the inner membrane of lysosome [11], and could activate DCs to induce humoral and cellular immunity [12-15]. It has been reported that CpG-ODN was the most effective immunostimulator at present, which was closely related to its structure [16-21]. Nevertheless, the electrostatic repulsion of free CPG-ODN resulted in low transmembrane transport efficiency and severely inhibited its immunomodulatory effect [22]. Therefore, it is urgent to develop suitable vector to delivery CpG-ODN in vivo for combined immunotherapy. Carbon dots (CDs) are emerging photoluminescence materials with many unique properties, including good biocompatibility, water solubility and easy functionalization [23,24]. Therefore, CDs are widely used in various drug delivery systems, bioactive compounds and gene delivery systems [25]. Our previous work had proved that hyaluronate (HA) and polyethylenimine (PEI) functionalized carbon dots (HP-CDs) displayed great potential in nucleic acid loading capacity for gene delivery [26]. Meanwhile, CDs with 
strong absorbance in the near infrared (NIR) region exhibited high photothermal conversion efficiency and might be used as ideal photosensitizer for PPT [27].

In addition, systemic using of immunotherapy drugs is difficult to avoid their non-specific biological distribution in normal tissues, while this nonspecific off-targeting of the immune system activates autoimmune-like damage to normal tissues. This phenomenon increases the risk of potential immunerelated adverse events (irAEs) while limiting the effectiveness of drugs [28, 29]. Recently, mounting evidences demonstrated that in situ treatment of tumor could avoid this risk. Injectable hydrogel is an excellent drug-loading platform, which has good biocompatibility and none-immunogenicity. It can be in a flowing state before injection and rapidly form gel network in situ after injection, which shows great potential in biomedical applications [30,31].

Inspired by the above statements, we aim to develop an in situ immunoinducible hydrogel based on the CDs for PTT and CpG-ODN delivery in vivo. Firstly, we prepared aluminum doped amino carbon quantum dots (A-CDs) using diethylenetriamine pentaacetic acid (DTPA) and the polyethyleneimine (PEI) as precursors. Then, the resultant A-CDs was modified by mannose for DCs targeting and loaded the CpGODNs to form the M/A-CDs@CpG. Finally, immunoinducible carbon dots-incorporated hydrogels (iCD@Gel) was fabricated through a dynamic covalent Schiff base reaction between amino groups of M/A-CDs@CpG and aldehyde groups of oxidized glucan [32]. We explored the phototermal performance and gene delivery capacity of M/A-CDs,as well as the impact of M/A-CDs@CpG treatment on the DCs maturation and antigen cross presentation in vitro. Using the M/A-CDs@CpG as cross-linker, we further studied that rheological properties of iCD@Gel. Afterward, we also demonstrated that the effect of on-site formation of iCD@Gel on the tumor growth and immune system via photothermal ablation and antitumor immune activation was investigated by $4 \mathrm{~T} 1$ tumor bearing mice. Especially, we focused on the the DCs maturation and cytotoxic $\mathrm{T}$ lymphocyte $\left(\mathrm{CD} 8^{+} \mathrm{T}\right)$ profiles in vivo after treatment.

\section{Methods}

\section{Materials}

Polyethyleneimine (PEI), Glucan, Mannose, Triacetylamino sodium borohydride, Aluminum chloride, and hexahydrate $\left(\mathrm{AlCl}_{3} \cdot 6 \mathrm{H}_{2} \mathrm{O}\right)$ were purchased from Aladdin Reagent Co.,Ltd. (Shanghai).

Diethylenetriaminepentacetic acid (DTPA), Sodium periodate are purchased from McLin Reagent Co.,Ltd. (Shanghai). Cell counting kit-8 (CCK-8) and 4,6-diamino-2-phenylindole (DAPI) were obtained from Thermo Fisher Scientific. Phosphate buffered saline (PBS) is purchased from Hyclone (Logan City, Utah, USA). Dulbecco minimum essential medium (DMEM ), RPMI modified medium ( RPMI-1640) and fetal bovine serum (FBS) were purchased from Hyclone (Logan, UT, USA). Trypsin was from Sigma-Aldrich (USA). Deionized water was used in the experiment.

\section{Synthesis of iCD@Gel}


$4 \mathrm{~g}$ of DTPA was dissolved in $30 \mathrm{ml}$ deionized water and mixed with PEl solution ( $12.5 \mathrm{~g}$ dissolved in 200 $\mathrm{ml}$ ). After microwave heating the supernatant was collected by centrifugation (3,000 r.p.m., 15 min). After dialysis CDs powder was obtained by freeze-drying. $4 \mathrm{~g}$ DTPA was dissolved in $30 \mathrm{ml}$ deionized water and mixed with $30 \mathrm{ml} \mathrm{AlCl} 3.6 \mathrm{H}_{2} \mathrm{O}$ solution $(0.1 \mathrm{~g} / \mathrm{mL}$ ) (solution 1). $200 \mathrm{ml}$ PEl solution was mixed with the solution and reacted at room temperature. After microwave heating for the supernatant was collected by centrifugation (3,000 r.p.m., $15 \mathrm{~min}$ ) after dialyzed and freeze-drying, A-CDs powder was obtained. ACDs powder was mixed with tracetamyl sodium borohydride and mannose at a mass ratio of $1: 1: 1$ to slow stirring at room temperature. After dialyzed and vacuum freeze-drying, M/A-CDs precipitation was obtained. M/A-CDs@CpG was obtained by mixing CpG with M/A-CDs powder at a mass ratio of $1: 100$. To prepare the oxidized glucan, $3.24 \mathrm{~g}$ of glucan and $8.65 \mathrm{~g}$ of sodium periodate were dissolved in $70 \mathrm{~mL}$ of deionized water with stirring in the dark room for $20 \mathrm{~min}$. Then, $1 \mathrm{~mL}$ of diethylene glycol was added to terminate the reaction. The obtained solution was dialyzed to obtain oxidized glucan powder by vacuum freeze-drying. To obtain the gel, $69.4 \mathrm{mg} \mathrm{M} / \mathrm{A}-\mathrm{CDs} @ \mathrm{CpG}$ was dissolved in $1 \mathrm{~mL}$ deionized water, and 80 mg oxidized dextran was dissolved in $1 \mathrm{~mL}$ deionized water, respectively. After mixing the two solutions, gel-likeiCD@Gel could be obtained immediately.

\section{Instruments and characterizations of iCD@Gel}

The phase structures were analyzed by X-ray diffractometer (XRD) on Rigaku SmartLab $9 \mathrm{~kW}$ with a Cu Ka radiation source $(\lambda=0.15418 \mathrm{~nm})$, a slit of 0.5 , and a scanning speed of $7 / \mathrm{min}$. X-ray photoelectron spectroscopy (XPS) analysis was performed on Thermo Fisher Nexsa (USA). A560 dual beam uv-visible spectrophotometer (AOE Instruments, Shanghai) was used to identify the uv-visible absorption spectrum. The morphology of M/A-CDs@CpG was observed by transmission electron microscope (TEM, FEI Talos F200S, America).

\section{Photothermal conversion efficiency}

The photothermal conversion efficiency of $\mathrm{M} / \mathrm{A}-\mathrm{CD}$ s is calculated according to the method previously reported [33]. Under the irradiation of $2.0 \mathrm{~W} / \mathrm{cm}^{2} 808 \mathrm{~nm}$ near-infrared laser, the temperature change within $600 \mathrm{~S}$ was recorded, and then the temperature decline process within $600 \mathrm{~S}$ was recorded after the laser was turned off. The photothermal conversion efficiency $(\eta)$ was calculated according to the following formula (1)-(3).

$$
\begin{gathered}
\eta=\frac{h S\left(\Delta T_{\max , \operatorname{mix}}-\Delta T_{\left.\max , H_{2} O\right)}\right.}{I\left(1-10^{-A_{800}}\right)} \\
\mathrm{hS}=\frac{\sum_{\mathrm{i}} \mathrm{m}_{1} \mathrm{C}_{\mathrm{p}, i}}{t} \ln \theta \\
\theta=\frac{\Delta \mathrm{T}}{\Delta \mathrm{T}_{\max , \operatorname{mix}}}
\end{gathered}
$$


In the above formula, $h$ is the heat transfer coefficient, $S$ is the surface area of the container, $\Delta T_{\text {max, mix }}$ is the temperature change of $\mathrm{A}-\mathrm{CD}$ s at the maximum stable temperature. $\Delta \mathrm{T}_{\max , \mathrm{H} 2 \mathrm{O}}$ is the temperature change of water at its maximum stable temperature. I is the laser power, and A808 is the absorbance value of $A-C D s$ in aqueous solution at $808 \mathrm{~nm}$ wavelength. $\theta$ is the dimensionless driving force temperature and is defined as the ratio of $\Delta \mathrm{T}$ to $\Delta \mathrm{T}_{\max }$ mix.

\section{Gel retardation experiments}

$2.0 \mathrm{~g}$ of agarose was weighed and placed in a triangular flask. $100 \mathrm{~mL} 1 \times$ TAE electrophoresis buffer was added and heated to complete dissolution in a microwave oven. When the solution temperature was cooled to about $50{ }^{\circ} \mathrm{C}, 1.1 \mu \mathrm{L}$ of GelRed dye solution was added to mix well. A sufficient amount of TAE buffer was added into the electrophoresis tank to make the gel immersed in the liquid at a depth of about $1 \mathrm{~cm}$. According to different mass ratio of CpG : M/A-CDs NPs $(1: 1,1: 2,1: 5,1: 10,1: 50,1: 100,1$ : $200,1: 500,1: 100)$ this two solutions were mixed into complex and $7 \mu$ solution, and then $1 \mu$ loading solution was added. The samples were carefully added to the gel sample hole with a liquid shifter, and 8 $\mu \mathrm{L}$ of sample was added to each hole. Then the current was switched on, the voltage was $100 \mathrm{~V}$. Stop electrophoresis about 30 minutes later and observe the fluorescent band under ultraviolet light.

\section{Hemolysis test}

After successful anesthesia, the mice's eyeballs were removed with forceps, and fresh blood from the eyeballs was collected into the heparin anticoagulant blood collection tube. The mouse red blood cells (MRBC) were collected after being cleaned for 3 times. The diluted MRBC $(100 \mu \mathrm{L})$ was co-incubated with $\operatorname{ACMC}(4,10,15,20,25,40,80,100 \mu \mathrm{g} / \mathrm{mL})$ at room temperature. PBS was set as negative control and deionized water as positive control. After 2-6 hours, centrifugation at 1000 RPM for 1 minute, the absorbance of supernatant was measured by a dual-beam UV-vis spectrophotometer.

\section{Biocompatibility of A-CDs}

CCK-8 method was used to evaluate the biocompatibility of A-CDs. Different concentrations of CDs ( 0.05 , $0.1,0.2,04,0.8,1,5,8,10 \mu \mathrm{g} / \mathrm{mL}$ ) were co-incubated with DC 2.4 cells and MEF cells (mouse embryonic fibroblast cell line) in 96 -well plates $\left(37^{\circ} \mathrm{C}, 5 \% \mathrm{CO}_{2}\right)$, about $1 \times 10^{4}$ cells per well. After $48 \mathrm{~h}$ incubation cells were incubated with $10 \mu \mathrm{L} \mathrm{CCK}-8$ reagent and $90 \mu \mathrm{L}$ DMEM medium. Then absorbance of the solution at $450 \mathrm{~nm}$ was measured by UV spectrophotometer (BioTek Instruments, Inc Vermont, USA). The experiment was repeated in three parallel groups. The relative cell viability was calculated as (Abs sample ${ }^{-}$ Abs $_{\text {zero-sit }}$ ) / (Abs control-Abs zero-sit $) \times 100 \%$.

\section{Phagocytosis experiment}

The process and distribution of M/A-CDs@CpG NPs in cells were studied by confocal laser scanning.4T1 cells were seeded at the bottom of the 24 -well plate $\left(10^{4}\right.$ cells/well in one pass, $37{ }^{\circ} \mathrm{C}$ and $5 \%$ carbon dioxide, $24 \mathrm{~h}$ ). When the cell density reached $60 \%$, M/A-CDs NPs were added and cultured for 30 min. 
After cleaning 3 times, the cells were fixed with $4 \%$ paraformaldehyde(room temperature, $15 \mathrm{~min}$ ) and the nuclei were stained with DAPI working solution in dark room for 5 min. After strong cleaning, the small disk was taken out for production. Sealed with neutral resin and stored at $4{ }^{\circ} \mathrm{C}$. Observe cells under laser confocal microscope.

\section{In vitro immune analysis}

DC 2.4 cells were seeded in 6-well plates $\left(1 \times 10^{6}\right.$ cells/well), cultured with different concentrations and different treatments of NPs at $37^{\circ} \mathrm{C}$ and $5 \% \mathrm{CO}_{2}$ to induce DC 2.4 cells maturation. After 48h, DC 2.4 cells were collected and stained with anti-CD86 PE, anti-CD80 APC, analyzed by flow cytometry. Another group of experiments, DC 2.4 cells and OVA + NPs co-culture, 48h after collecting DC 2.4 cells stained with $\mathrm{H} 2 \mathrm{~kb}-\mathrm{PE}$, flow cytometry analysis results.

\section{Animals}

Female Babl/c mice (18-22 g, 6-8 weeks old) were purchased from Changzhou Cavents Laboratory Animal Co., Ltd. The housing conditions for the animals with an ambient temperature of $24{ }^{\circ} \mathrm{C}$ and a relative humidity of $50 \%$.All animal operations in accordance with Jiangsu University Laboratory Animal Care and Use Guide.The $4 \mathrm{t} 1$ tumor mice treated with drugs were divided into six groups, including PBS, CDs NPs, A-CDs NPs, M/A-CDs, M/A-CDs@CpG NPs and iCD@Gel. When the average size of the tumor reached about $100 \mathrm{~m} 3$, nano-drugs were injected in situ. (1 $\mathrm{mg} / \mathrm{mouse}$ in treatment group), hydrogel group was injected with double-channel syringe. The tumors were irradiated by $808 \mathrm{~nm}$ laser with a power density of $2 \mathrm{~W} / \mathrm{cm}^{2}$ for 5 minutes on the $1 \mathrm{st}$, 3rd and 5th day, respectively. The tumor volume was monitored with vernier caliper every 2 days during treatment for 20 days. For immunological analysis, inguinal lymph nodes and spleens were isolated and made into single-cell suspensions after 7 days of treatment to 4T1 tumor mice. Inguinal lymph node cells suspensions and spleen cells suspensions were stained with APC anti-mouse CD3 (BioLegend), PE anti-mouse CD8a (BioLegend), APC anti-mouse CD11C, FITC anti-mouse CD80, PE anti-mouse CD86, respectively. The proportion of CD11 $\mathrm{C}^{+} \mathrm{CD} 80^{+} \mathrm{CD} 86^{+}$ cells and $\mathrm{CD} 3^{+} \mathrm{CD} 8^{+}$corresponding cells in DCs and effector memory T cells.

\section{Histocompatibility}

In order to study biocompatibility, the main organs (heart, spleen, liver, lung, kidney) of mice in different treatment groups were collected. Soak in $4 \%$ paraformaldehyde buffer solution, then trim, dehydration, transparent treatment, paraffin embedding.Specimens were sliced continuously, thickness $5 \mu \mathrm{m}$, hematoxylin and eosin staining. Tissue sections were observed under an optical microscope.

\section{Statistical analysis}

Each experiment was repeated at least three times independently. All data are represented by mean standard deviation. Student $t$ test was used for statistical analysis of the two groups, and single factor analysis of variance was used for difference between groups. P value was calculated by GraphPad Prism 
8 software. The significant difference was expressed as ${ }^{*} P<0.05,{ }^{*} P<0.01,{ }^{*} * P<0.001$ and $\star * * * P<0.0001$.

\section{Results And Discussion}

\section{Preparation and characterization of the iCD@Gel}

The procedure for the synthesis of iCD@Gel was illustrated in scheme 1. Briefly, the aluminum doped carbon dots (A-CDs) were synthesized by hydrothermal carbonization using PEl as passivator and metal chelating agent DTPA. Then, these A-CDs were surface modified by mannose as a targeting agent via chemical grafting to form M/A-CDs. Next, the M/A-CDs could be used as nanocarrier for loading immune adjuvant CpG ODNs by electrostatic adsorption to synthesize M/A-CDs@CpG. Finally, iCD@Gel was fabricated by the Schiff base reaction between amino groups on the M/A-CDs and aldehyde groups on the oxidized glucan.

The typical transmission electron microscopy (TEM) images showed that the resultant M/A-CDs were spherical with an average diameter of approximate $4.3 \mathrm{~nm}$ (Fig. 1a and b). This size allowed them to stay in the blood circulation for a long time and passively accumulate in tumors through enhanced permeability and retention (EPR) effects [34]. It could be found from the X-ray diffraction pattern in Fig. 1c that a wide diffraction peak appeared at 25, which proved that M/A-CDs has amorphous phase structure. The chemical composition of M/A-CDs was characterized by FTIR and XPS. The FTIR spectra of M/ACDs showed (Fig. 1d) that a wide absorption peak appeared near $3300 \mathrm{~cm}^{-1}$, which was ascribed to $-\mathrm{OH}$ stretching vibration. The asymmetric stretching vibration peak of $-\mathrm{CH}_{2}$ appeared near $2926 \mathrm{~cm}^{-1}$, and the peak at $1613 \mathrm{~cm}^{-1}$ belonged to the scissors bending vibration of $-\mathrm{NH} 2$. Two small and sharp peaks at $1460 \mathrm{~cm}^{-1}$ and $1465 \mathrm{~cm}^{-1}$ belonged to the antisymmetric bending vibration peak of $-\mathrm{CH}_{3}$ groups. The peak near $1283 \mathrm{~cm}^{-1}$ corresponded to the bending vibration signal frequency of $-\mathrm{CH}$, and the peak at $1092 \mathrm{~cm}^{-1}$ corresponded to the vibration contraction of $-\mathrm{C}-\mathrm{O}$. These results showed tha there are many hydriphilic groups on the surface of M/A-CDs, which endowed M/A-CDs with superior stability and dispersion in water. In addition, the introduction of nitrogen element could significantly improved the luminescence intensity, that was meaningful to the multifunctional applications in bioimaging. The full XPS spectra in Fig.1e showed three typical peaks, C1s (284.55 eV), N1s (398.62 eV) and 01s (531.64 eV) with the contents of $77.91 \%(\mathrm{C}), 10.8 \%(\mathrm{~N})$ and $10.79 \%(0)$, respectively. The atomic ratios of N/C and O/C were $44.6 \%$ and $83.22 \%$, (Fig. $1 \mathrm{f}-\mathrm{i}$ ), indicating that A-CDs were highly doped with the element of $\mathrm{N}$ and $\mathrm{O}$. At the same time, the existence of aluminum was verified by two small peaks at $84.98 \mathrm{eV}-65.18 \mathrm{eV}$, and the content was $0.5 \%$. This result proved that aluminum has been doped into the CDs in an appropriate proportion. To verify the grafting of mannose, the nuclear magnetic hydrogen spectrum of M/A-CDs was detected. It could be seen (Fig. 2a) that the same shape peak appeared at the same position in the nuclear magnetic hydrogen spectrum of M/A-CDs sample and pure mannose, which proved that the mannose was successfully grafted to the surface of $A-C D s$. 
The gene loading capacity of M/A-CDs was evaluated using gel retardation assay. We envisioned that CpG ODNs with negatively charge could move to the negative electrode in agarose gel electrophoresis, but this migration could be reversed when the charge was neutralized by M/A-CDs (Fig. 2b). The results (Fig. 2c) showed that the CpG ODNs bands could still be observed clearly in the positive electrode when the mass ratio of $\mathrm{CPG}$ ODNs: M/A-CDs was less than 1:5. However, the bands became lighter when the mass ratio decreased to 1:10, indicating that only a small amount of $\mathrm{CPG}$ ODNs bound with M/A-CDs and failed to migrate. When the mass ratio decreased to $1: 50, \mathrm{CpG}$ ODNs bands were completely blocked to the opposite direction of migration. The tendency became strong with the decreasing mass ratio.

In Fig.2d, the results showed that zeta potential of CpG ODNs was $-29.2 \mathrm{mV}$ and zeta potential of M/ACDs was $27.9 \mathrm{mV}$. Whereas zeta potential of CpG ODNs mixed with M/A-CDs was $11.7 \mathrm{mV}$, which further confirmed M/A-CDs bound with CpG ODNs. These results proved that the M/A-CDs possessed the ability to carry the $\mathrm{CpG}$ ODNs for targeted delivery.

Hydrogel itself as ideal delivery mode has profound impacts on the efficiency of immune activation [35]. Therefore, we attempted to develop an in situ hydrogel using the M/A-CDs as cross-liker to further enhance their immune response. As we expected, the amino groups on the surface of M/A-CDs and the aldehyde group in side chain of dextran underwent aldoamine condensation via the schiff base reaction, leading to the rapid formation of hydrogel (iCD@Gel) in the Fig. 2e. The morphology of hydrogel was observed by SEM (Fig. 2f), it was found that iCD@Gel had a typical sponge scaffold appearance. This dense network structure plays an important role in the performance of hydrogels and biomedical engineering applications. The internal interaction of hydrogels is one of the key factors affecting cell growth and drug release [36]. Afterward, the rheological properties of iCD@Gel was generally characterized by Young's modulus. The amplitude scan (Fig. $\mathbf{2 g}$ ) shown the dependence of storage modulus G' and loss modulus G" of iCD@Gel on the applied strain ( $\gamma$ \%). The result indicated that the storage modulus $\mathrm{G}^{\prime}$ was significantly higher than the loss modulus $\mathrm{G}$ " in a large range, showing that iCD@Gel possessed a gel organization. In addition, iCD@Gel had elasticity up to 12010 \% strain at a fixed angular frequency of $1 \mathrm{~Hz}$ before the gels collapsed (G"> G'). which proved that the iCD@Gel had extraordinary mechanical properties. The extraordinary mechanical strength may be ascribed to the contribution of the imine bond network within the iCD@Gel [37-39].

\section{Optical characteristics of M/A-CDs}

Given the intrinsic property of CDs, the optical characteristics of M/A-CDs was analyzed by UV-Vis spectrum and PL spectrum (Fig. 3a). It can be seen from Fig. 3c that the PL emission of M/A-CDs reached a peak at $462.9 \mathrm{~nm}$ under the $346 \mathrm{~nm}$ excitation wavelength. When excitation wavelength increased from $300 \mathrm{~nm}$ to $500 \mathrm{~nm}$, the emission had a wide range from $450 \mathrm{~nm}$ to $650 \mathrm{~nm}$, the red shift was obvious due to the photon reabsorption (Fig. 3b). These results proved that the M/A-CDs we prepared were different from traditional fluorescent substances beacuse they had excitation-lightdependent emission characteristics. In the UV absorption spectra of M/A-CDs (Fig. 3d), there was strong optical absorption in the ultraviolet region due to the $\pi-\pi^{\star}$ transition of the $C=C$ bond on their surface. The maximum absorption peak at about $320 \mathrm{~nm}$ and extending to the visible region. Meanwhile, M/A- 
CDs showed bright blue fluorescence under UV light irradiation, which verified their favorable fluorescence performance.

\section{Photothermal property of M/A-CDs}

Mounting evidence demonstrated that CDs exhibited favorable photothermal behavior due to the nonspecific absorption in near infrared region, but their photothermal conversion efficiency (PCE) were inefficient. Therefore, we tended to improve the photothermal performance of CDs through heteratomic doping strategy. The results shown that the PCE of M/A-CDs was higher than traditional CDs, indicating that aluminum doping could significantly promote the PCE of CDs in Fig. 4a. Based on the calculation formula form previously publications [40], the photothermal conversion efficiency of M/A-CDs was 37.7 $\%$, which was much higher than those of bare CDs without aluminum ions (13.3 \%) (Fig. 4b-e). These findings shown that the incorporation of metal elements could enhance the photothermal effect of CDs significantly. This phenomenon may be attributed to the doping of metal elements producing a new electron transition path inside the CDs, thereby enhancing the radiation heat efficiency [41]. Subsequently, the photothermal performance of $\mathrm{M} / \mathrm{A}-\mathrm{CD}$ in aqueous solution $(1.5 \mathrm{mg} / \mathrm{mL})$ was studied under different power densities. As shown in Fig. 4f, the temperature of M/A-CDs solution increased sharply with the laser power increased. It was reported that hyperthermal could induce intense apoptosis of tumor cells when the temperature reached $55^{\circ} \mathrm{C}$. Under the same laser power $\left(2 \mathrm{~W} / \mathrm{cm}^{2}\right)$, different concentrations of M/A-CDs irradiation solution also showed excellent photothermal effect (Fig. $\mathbf{4 g}$ ). During this period, the heating imaging of M/A-CDs was recorded by the infrared camera, which was consistent with temperature curve (Fig. 4h). These results showed that the M/A-CDs could convert the NIR laser energy into thermal energy effectively in a concentration-dependent manner. As shown in Fig. $4 \mathbf{i}$, the temperature curve of the traditional photosensitizer indocyanine green (ICG) gradually decreased after the five laser on/off cycles. In contrast, the temperature curves of M/A-CDs remained during same time, which indicating that M/A-CDs has excellent photothermal stability. Besides, the introduction of aluminum ions may be used as tranditional immune adjuvant and facilitate anti-tumor immune activation.

\section{Biocompability, intracellualr uptake and photothermal ablation of M/A-CDs}

The biocompability characterization of biomaterials is very essential before biomedical applitions. As shown in Fig. 5a and b, the presence of two absorption peaks at $560 \mathrm{~nm}$ after water treatment indicated hemolysis. On the contrary, different concentrations of M/A-CDs had little effect on the blood cells. The cytotoxicity of M/A-CDs in vitro was detected by standard CCK-8 method. The DC 2.4 and MEF cells were co-cultured with M/A-CDs at different concentrations for $48 \mathrm{~h}$, respectively. The result shown that both of cells viability were maintained above $95 \%$ after treatment, indicating the favorable cytocompability of M/A-CDs (Fig. 5c). Then, M/A-CDs was evaluated as a photothermal agent for ablation of cancer cells in vitro under laser irradiation. 4T1 cells were exposed to $1.5 \mathrm{~W} / \mathrm{cm}^{2}$ power density of $808 \mathrm{~nm}$ laser after incubation with different concentrations of M/A-CDs for $4 \mathrm{~h}$. The results showed that cells viability obviously decreased with the increase of M/A-CDs concentration under NIR laser irradiation (Fig. $\mathbf{5 d ) . ~ T a k e n ~ t o g e t h e r , ~ t h e s e ~ r e s u l t s ~ c l e a r l y ~ v e r i f i e d ~ t h a t ~ M / A - C D s ~ p o s s e s s e d ~ e x c e l l e n t ~ b i o c o m p a t i b i l i t y ~ a n d ~}$ 
photothermal performance of promoting tumor cells ablation in vitro. The phagocytosis and subcellular localization of M/A-CDs in 4T1 cells were observed using different organelle probes by confocal fluorescence microscopy. As shown in Fig. 5e, the green fluorescence derived from M/A-CDs could be observed in the cytoplasm after co-cultured for $4 \mathrm{~h}$. After being labelled by different organelle probes, the strong orange-yellow fluorescence could be observed in lysosomes and mitochondria. This preference distribution may be ascribed to positive charge nature of M/A-CDs.

\section{Immune activation function of M/A-CDs@CpG in vitro}

DCs with powerful antigen-presenting ability play key role in anti-tumor immunity [42]. Hence, we studied the effect of M/A-CDs@CpG on the maturation and antigen-presenting ability of DCs according to the principle in Fig. 6a. Initially, the CD80 and CD86 expression of DCs in the A-CDs group (11.4\%) was 2.5 times higher than control group (4.5\%) in the Fig. $\mathbf{6 b}$ and c. Notably, the CD80 and CD86 expression of DCs in the M/A-CDs@CpG group was up to 17.8\%, indicating that the M/A-CDs@CpG possessed a strong ability to induce DCs maturation. Meanwhile, the CD80 and CD86 expression increased with the increase of M/A-CDs@CpG concentration from 6.3 \% to 19.8 \% (Fig. 6d and e), further confirming the above results. It is well known that antigen presentation by classical MHC class I molecules to $\mathrm{CD} 8^{+} \mathrm{T}$ cells is a central aspect of the adaptive immune response. Hence, we choose SIINFEKL peptides derived from OVA as model molecules to exploit the antigen cross presentation ability of DCs. As shown in Fig. $\mathbf{6} \mathbf{f}$ and $\mathbf{g}$, the H-2Kb-SIINFEKL peptide expression (32.4 \%) in M/A-CDs@CpG group could be detected by flow cytometry, which was higher than that in control group (17.9\%), CDs group (24.5\%), A-CDs group (25.2 \%), M/A-CDs group (28.4\%). It could be seen that M/A-CDs@CpG exhibited the strong ability to promote antigen cross-presentation performance of DCs.

\section{The anti-tumor effect of iCD @Gel in vivo}

Encouraged by the excellent PPT and immune activation performance in vitro, we further explored the inhibition of iCD@Gel on tumor through tumor-bearing mice (Fig. 7a). Upon 808 nm laser irradiation, the tumor temperature in all treatment groups increased rapidly after 5 mins except PBS group (Fig. $7 \mathrm{~b}$ and c). It was worth noting that A-CDs exhibited higher photothermal performance than CDs, which was ascribed to introduction of alumimum. Among them, iCD@Gel not only raised the temperature of tumors from $26^{\circ} \mathrm{C}$ to $56^{\circ} \mathrm{C}$ within $5 \mathrm{~min}$, but also stayed inside the tumor for up to 5 days (Fig. 7d). These results proved that the iCD@Gel possessed superior photothermal performance and long term retention in vivo, which was conductive to extend therapeutic window and persistent immunostimulation.

Subsequently, we investigated the therapeutic effect of iCD@Gel using the 4T1 tumor-bearing mice. The results shown that CDs-mediated PTT in the CDs, A-CDs, M/A-CDs, M/A-CDs@CpG, iCD@Gel groups significantly suppressed the growth of tumors, compared with that in PBS group. Impressively, the tumor in the iCD@Gel group was almost eradicated and did not relapse within 20 days (Fig. 7e and f). The survival time of mice in iCD@Gel group was extended by more than 60 days (Fig. 7g). In addition, weight fluctuations in all groups throughout the treatment process could be negligible, which confirmed that 
these biomaterials have no significant adverse effects on mice (Fig. 7h). At last, we assessed the longterm potential biocompatibility by the histopathological section when the experiments was terminated. It was apparently that no pathological changes or inflammatory could be observed in major organs (Fig. 7i). These results indicated that iCD@Gel with high phototermal performance has potential to serve as a novel therapeutic method in precision therapy of tumor in situ.

\section{The anti-tumor immune activation of iCD@Gel in vivo}

The strong anti-tumor immune activation is essential to prevent tumor recurrence and metastasis. Therefore, the anti-tumor immune activation of the iCD@Gel in vivo were evaluated through analysis of the DCs maturation and $\mathrm{CD} 8^{+}$cytotoxic T lymphocytes (CTLs) differetiation. After distinct treatments, the lymph nodes and spleen tissues were collected for flow cytometry analysis. Consistent with the results in vitro, the iCD@Gel triggered the higher expression level of $C D 80^{+} / C D 86^{+} D C s$ than the other treatments (Fig. 8a). Specifically, the ratio of $C D 80^{+} C D 86^{+}$DCs in the lymph nodes in PBS, CDs, A-CDs, M/A-CDs, M/A-CDs@CpG, iCD@Gel treatment were 1.6 \%, 2.8 \%, 4.4 \%, 10.8 \%, 15 \% (Fig. 8b). The ratio of $\mathrm{CD} 80^{+} \mathrm{CD} 86^{+} \mathrm{DCs}$ in the spleen in different treatments gradually increased from $0.7 \%$ to $5 \%$ (Fig. $8 \mathrm{e}$ ), which was similar to the trend in lymph nodes. These phenomenons may be ascribed to the PTT-triggered DAMPs release and CpG ODNs stimulation. Generally, the iCD@Gel exhibited the outstanding ability to induce DCs maturation. Afterward, the $\mathrm{CD} 8^{+}$cytotoxic $\mathrm{T}$ cells in the lymph nodes was measured after treatments. The results shown that the ratio of $C D 8^{+} \mathrm{T}$ cells in the lymph nodes in PBS, CDs, A-CDs, M/ACDs, M/A-CDs@CpG, iCD@Gel treatment was 24.7 \%, 31.7 \%, 33.5 \%, 39 \%, 42.2 \% and 48.8 \% (Fig. 8f and g). Compared with the treatment groups that free of hydrogels, iCD@Gel induced more higher frequency of $\mathrm{CD}^{+} \mathrm{T}$ cells, indicating positive effect of hydrogels to the immune activation. The identical trend of percentage of $\mathrm{CD}^{+} \mathrm{T}$ cells in spleens could be observed from $14 \%$ to $41.5 \%$ (Fig. $8 \mathrm{~h}$ and i). All of these results demonstrated that iCD@Gel significantly increased the frequency of $C D 8^{+} T$ cells in the spleen and the lymph nodes, which was conducive to anti-tumor immune response.

\section{Conclusion}

In this work, we developed a novel in situ iCD@Gel for combined photothermal immunotherapy of cancer. The resultant iCD@Gel was composed of versatile M/A-CDs and aldehyde glucan. It was found that the introduction of aluminum into M/A-CDs greatly improved their photothermal conversion efficiency (37.7\%), which was higher than traditional CDs (13.3\%). In addition, M/A-CDs with mannose modification could be served as nanocarrier to load CpG ODNs for inducing the maturation of DCs via manose receptor-mediated targeting delivery. Upon intratumoral injection, the iCD@Gel was in situ formed instantly via the M/A-CDs cross-linked aldehyde glucan based on Schiff base reaction. As we expected, the iCD@Gel induced immunogentic tumor cell death and DAMPs release via photothermal ablation under 808 nm NIR irradiation. More importantly, the iCD@Gel synergized with the DAMPs to significantly promote the maturation and antigen cross-presentation of DCs. These results indicated that the iCD@Gel 
has the potential to integrate photothermal ablation and anti-tumor immune activation for individualized precision therapy of tumor.

\section{Declarations}

\section{Ethics approval and consent to participate}

All experimental procedures were approved by the Animal Care and Use Committee of Medical College of Jiangsu University (No. UJS-IACUC-AP-20190314002).

\section{Consent for publication}

Each co-author has read the manuscript and approved its submission, and the work will be submitted to your journal.

\section{Availability of data and materials}

All data generated or analysed during this study are included in this published article.

\section{Competing interests}

The authors declare that they have no competing interests.

\section{Funding}

This work was supported by Natural Science Foundation of Jiangsu Province (SBK2020022937), and Zhenjiang key research and development program-social development (SH2019002), Major natural science research projects of colleges and universities in jiangsu province (19KJA150004), Changzhou Sci\&Tech Program (CJ20200108).

\section{Authors' contributions}

$\mathrm{SL}$, one of the main authors, was responsible for the preparation and characterization of nanomaterials. $\mathrm{JL}$ was in charge of cell culture. XX, HW and YW assisted in cell experiment and flow cytometry. M and QL assisted in the animal experiments. All authors read and approved the final manuscript.

\section{Acknowledgements}

The Acknowledged are included within the article.

\section{References}

1. Loo C, Lowery A, Halas N, West J, Drezek R. Immunotargeted nanoshells for integrated cancer imaging and therapy. Nano Lett. 2005;5(4):709-11. 
2. Huang $\mathrm{X}$, Jain PK, El-Sayed IH, El-Sayed MA. Plasmonic photothermal therapy (PPTT) using gold nanoparticles. Lasers Med Sci. 2008;23(3):217-28.

3. Sweeney EE, Cano-Mejia J, Fernandes R. Photothermal Therapy Generates a Thermal Window of Immunogenic Cell Death in Neuroblastoma. Small. 2018;14(20):e1800678.

4. Peng, X., Liu, Y., Bentley, W. E., \& Payne, G. F. Electrochemical Fabrication of Functional Gelatin-Based Bioelectronic Interface. Biomacromolecules, 2016;17(2):558-563.

5. Zhou F, Feng B, Yu H, Wang D, Wang T, Ma Y, Wang S, Li Y. Tumor Microenvironment-Activatable Prodrug Vesicles for Nanoenabled Cancer Chemoimmunotherapy Combining Immunogenic Cell Death Induction and CD47 Blockade. Adv Mater. 2019;31(14):e1805888.

6. Krysko DV, Garg AD, Kaczmarek A, Krysko O, Agostinis P, Vandenabeele P. Immunogenic cell death and DAMPs in cancer therapy. Nat Rev Cancer. 2012;12(12):860-75.

7. Fumet JD, Limagne E, Thibaudin M, Ghiringhelli F. Immunogenic Cell Death and Elimination of Immunosuppressive Cells: A Double-Edged Sword of Chemotherapy. Cancers (Basel). 2020;12(9):2637.

8. Vermaelen K. Vaccine Strategies to Improve Anti-cancer Cellular Immune Responses. Front Immunol. 2019;10:8.

9. Banstola A, Jeong JH, Yook S. Immunoadjuvants for cancer immunotherapy: A review of recent developments. Acta Biomater. 2020;114:16-30.

10. Y. Liu, B.M. Crawford, T. Vo-Dinh, Gold nanoparticles-mediated photothermal therapy and immunotherapy, Immunotherapy 2018;10(13):1175-1188.

11. Lu W, Cui C, Wang Y, Sun X, Wang S, Yang M, Yu Y, Wang L. CpG ODN as an adjuvant arouses the vigor of $B$ cells by relieving the negative regulation of surface TLR9 to enhance the antibody response to vaccine. Appl Microbiol Biotechnol. 2021;105(10):4213-4224.

12. Jia X, Guo J, Guo S, Zhao T, Liu X, Cheng C, Wang L, Zhang B, Meng C, Jia H, Luo E. Antitumor effects and mechanisms of $\mathrm{CpG}$ ODN combined with attenuated Salmonella-delivered siRNAs against PD-1. Int Immunopharmacol. 2021;90:107052.

13. Zhao Y, Wang H, Yang Y, Jia W, Su T, Che Y, Feng Y, Yuan X, Wang X. Mannose-Modified Liposome Co-Delivery of Human Papillomavirus Type 16 E7 Peptide and CpG Oligodeoxynucleotide Adjuvant Enhances Antitumor Activity Against Established Large TC-1 Grafted Tumors in Mice. Int J Nanomedicine. 2020;15:9571-9586.

14. Suo J, Yang Y, Che Y, Chen C, Lv X, Wang X. Anti-pulmonary metastases from cervical cancer responses induced by a human papillomavirus peptide vaccine adjuvanted with CpGoligodeoxynucleotides in vivo. Int Immunopharmacol. 2021;90:107203.

15. Valmori D, Souleimanian NE, Tosello V, Bhardwaj N, Adams S, O'Neill D, Pavlick A, Escalon JB, Cruz CM, Angiulli A, Angiulli F, Mears G, Vogel SM, Pan L, Jungbluth AA, Hoffmann EW, Venhaus R, Ritter G, Old LJ, Ayyoub M. Vaccination with NY-ESO-1 protein and CpG in Montanide induces integrated antibody/Th1 responses and CD8 T cells through cross-priming. Proc Natl Acad Sci U S A. 2007;104(21):8947-52. 
16. Hartmann G, Weiner GJ, Krieg AM. CpG DNA: a potent signal for growth, activation, and maturation of human dendritic cells. Proc Natl Acad Sci U S A. 1999;96(16):9305-10.

17. Zhu G, Liu Y, Yang X, Kim YH, Zhang H, Jia R, Liao HS, Jin A, Lin J, Aronova M, Leapman R, Nie Z, Niu $G$, Chen X. DNA-inorganic hybrid nanovaccine for cancer immunotherapy. Nanoscale. 2016;8(12):6684-92.

18. Speiser DE, Liénard D, Rufer N, Rubio-Godoy V, Rimoldi D, Lejeune F, Krieg AM, Cerottini JC, Romero P. Rapid and strong human CD8+ T cell responses to vaccination with peptide, IFA, and CpG oligodeoxynucleotide 7909. J Clin Invest. 2005;115(3):739-46.

19. Fourcade J, Kudela P, Andrade Filho PA, Janjic B, Land SR, Sander C, Krieg A, Donnenberg A, Shen H, Kirkwood JM, Zarour HM. Immunization with analog peptide in combination with $\mathrm{CpG}$ and montanide expands tumor antigen-specific CD8+ T cells in melanoma patients. $J$ Immunother. 2008t;31(8):781-91.

20. Katsuda M, Iwahashi M, Matsuda K, Miyazawa M, Nakamori M, Nakamura M, Ojima T, lida T, Hayata $\mathrm{K}$, Yamaue $\mathrm{H}$. Comparison of different classes of CpG-ODN in augmenting the generation of human epitope peptide-specific CTLs. Int J Oncol. 2011;39(5):1295-302.

21. Chen W, Jiang M, Yu W, Xu Z, Liu X, Jia Q, Guan X, Zhang W. CpG-Based Nanovaccines for Cancer Immunotherapy. Int J Nanomedicine. 2021;16:5281-5299.

22. Ming J, Zhang J, Shi Y, Yang W, Li J, Sun D, Xiang S, Chen X, Chen L, Zheng N. A trustworthy CpG nanoplatform for highly safe and efficient cancer photothermal combined immunotherapy. Nanoscale. 2020;12(6):3916-3930.

23. Xu A, Wang G, Li Y, Dong H, Yang S, He P, Ding G. Carbon-Based Quantum Dots with Solid-State Photoluminescent: Mechanism, Implementation, and Application. Small. 2020 ;16(48):e2004621.

24. Su Y, Liu S, Guan Y, Xie Z, Zheng M, Jing X. Renal clearable Hafnium-doped carbon dots for CT/Fluorescence imaging of orthotopic liver cancer. Biomaterials. 2020;255:120110.

25. Li X, Vinothini K, Ramesh T, Rajan M, Ramu A. Combined photodynamic-chemotherapy investigation of cancer cells using carbon quantum dot-based drug carrier system. Drug Deliv. 2020;27(1):791804.

26. M. Zhang, X. Zhao, Z. Fang, Y. Niu, J. Lou, Y. Wu, S. Zou, S. Xia, M. Sun, F. Du, Fabrication of HA/PEIfunctionalized carbon dots for tumor targeting, intracellular imaging and gene delivery, RSC Advances 2017;7(6):3369-3375.

27. B. Geng, D. Yang, D. Pan, L. Wang, F. Zheng, W. Shen, C. Zhang, X. Li, NIR-responsive carbon dots for efficient photothermal cancer therapy at low power densities, Carbon 2018;134:153-162.

28. Li J, Yu X, Jiang Y, He S, Zhang Y, Luo Y, Pu K. Second Near-Infrared Photothermal Semiconducting Polymer Nanoadjuvant for Enhanced Cancer Immunotherapy. Adv Mater. 2021;33(4):e2003458.

29. Kobayashi H, Choyke PL. Near-Infrared Photoimmunotherapy of Cancer. Acc Chem Res. 2019;52(8):2332-2339.

30. Chen Q, Wang C, Zhang X, Chen G, Hu Q, Li H, Wang J, Wen D, Zhang Y, Lu Y, Yang G, Jiang C, Wang J, Dotti G, Gu Z. In situ sprayed bioresponsive immunotherapeutic gel for post-surgical cancer 
treatment. Nat Nanotechnol. 2019;14(1):89-97.

31. Zhang J, Chen C, Li A, Jing W, Sun P, Huang X, Liu Y, Zhang S, Du W, Zhang R, Liu Y, Gong A, Wu J, Jiang $X$. Immunostimulant hydrogel for the inhibition of malignant glioma relapse post-resection. Nat Nanotechnol. 2021;16(5):538-548.

32. McKay CS, Finn MG. Click chemistry in complex mixtures: bioorthogonal bioconjugation. Chem Biol. $2014 ; 21(9): 1075-101$.

33. Lin H, Gao S, Dai C, Chen Y, Shi J. A Two-Dimensional Biodegradable Niobium Carbide (MXene) for Photothermal Tumor Eradication in NIR-I and NIR-II Biowindows. J Am Chem Soc. 2017;139(45):16235-16247.

34. Zhu M, Nie G, Meng H, Xia T, Nel A, Zhao Y. Physicochemical properties determine nanomaterial cellular uptake, transport, and fate. Acc Chem Res. 2013;46(3):622-31.

35. X. Peng, Y. Liu, W.E. Bentley, G.F. Payne, Electrochemical Fabrication of Functional Gelatin-Based Bioelectronic Interface, Biomacromolecules 2016;17(2):558-63.

36. J. Qu, X. Zhao, Y. Liang, T. Zhang, P.X. Ma, B. Guo, Antibacterial adhesive injectable hydrogels with rapid self-healing, extensibility and compressibility as wound dressing for joints skin wound healing, Biomaterials 2018;183:185-199.

37. Nam S, Stowers R, Lou J, Xia Y, Chaudhuri O. Varying PEG density to control stress relaxation in alginate-PEG hydrogels for 3D cell culture studies. Biomaterials. 2019;200:15-24.

38. Peng X, Liu Y, Bentley WE, Payne GF. Electrochemical Fabrication of Functional Gelatin-Based Bioelectronic Interface. Biomacromolecules. 2016;17(2):558-63.

39. Qu J, Zhao X, Liang Y, Zhang T, Ma PX, Guo B. Antibacterial adhesive injectable hydrogels with rapid self-healing, extensibility and compressibility as wound dressing for joints skin wound healing. Biomaterials. 2018;183:185-199.

40. Lin H, Gao S, Dai C, Chen Y, Shi J. A Two-Dimensional Biodegradable Niobium Carbide (MXene) for Photothermal Tumor Eradication in NIR-I and NIR-II Biowindows. J Am Chem Soc. 2017;139(45):16235-16247.

41. Li ZH, Chen Y, Sun Y, Zhang XZ. Platinum-Doped Prussian Blue Nanozymes for Multiwavelength Bioimaging Guided Photothermal Therapy of Tumor and Anti-Inflammation. ACS Nano. 2021;15(3):5189-5200.

42. Veglia F, Gabrilovich DI. Dendritic cells in cancer: the role revisited. Curr Opin Immunol. 2017;45:4351.

\section{Figures}


a

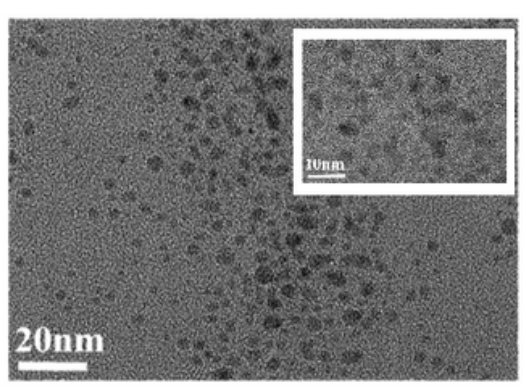

d
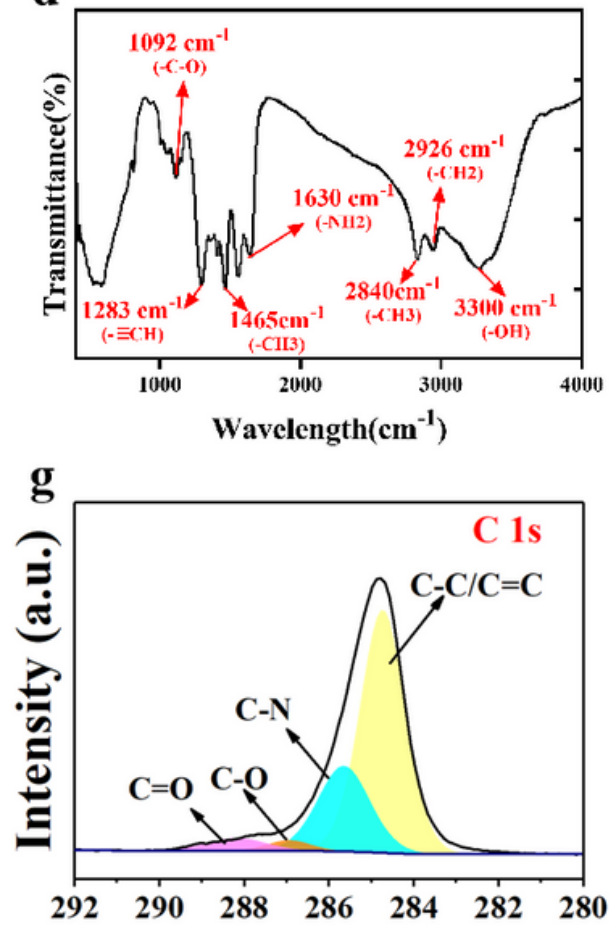

Binding Energy (eV)
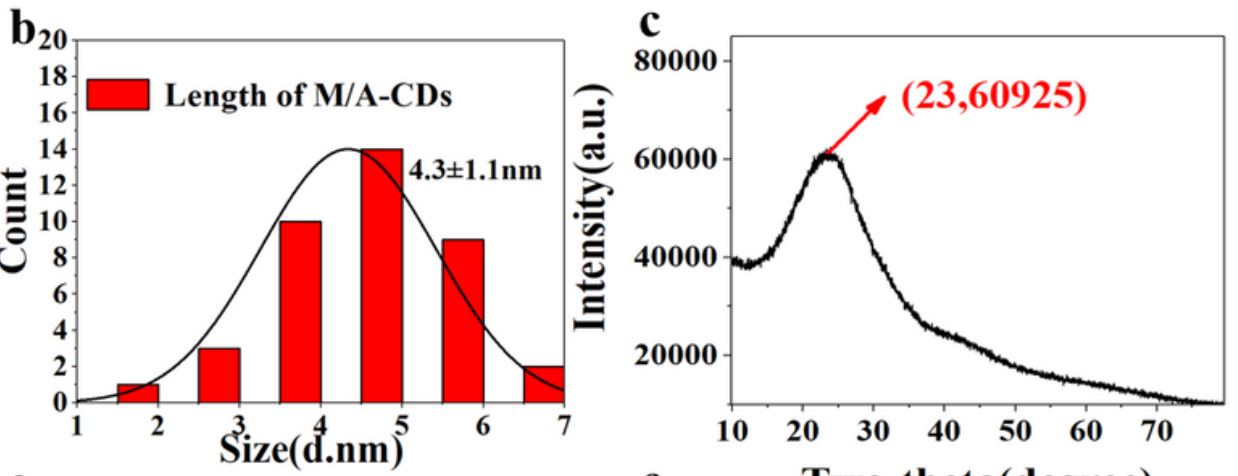

e

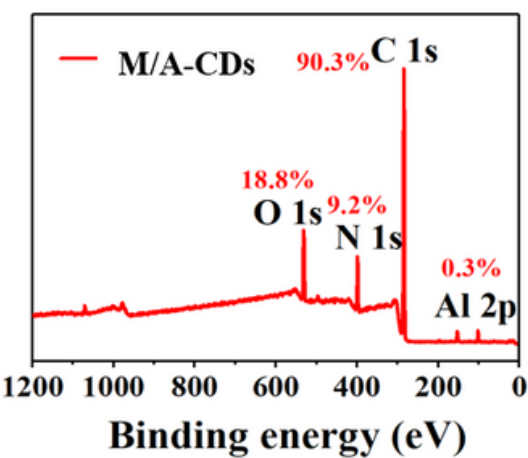

h

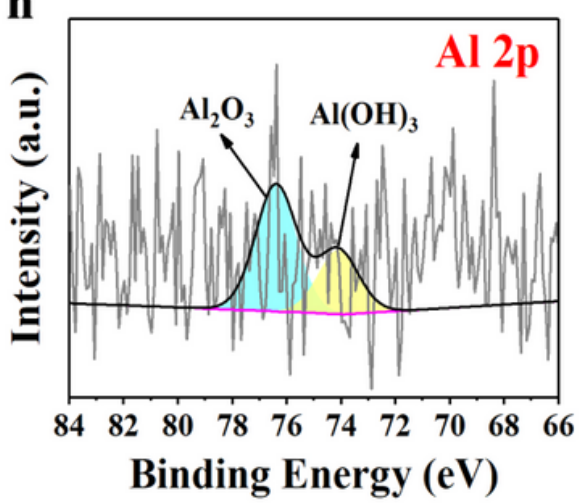

f Two-theta(degree)
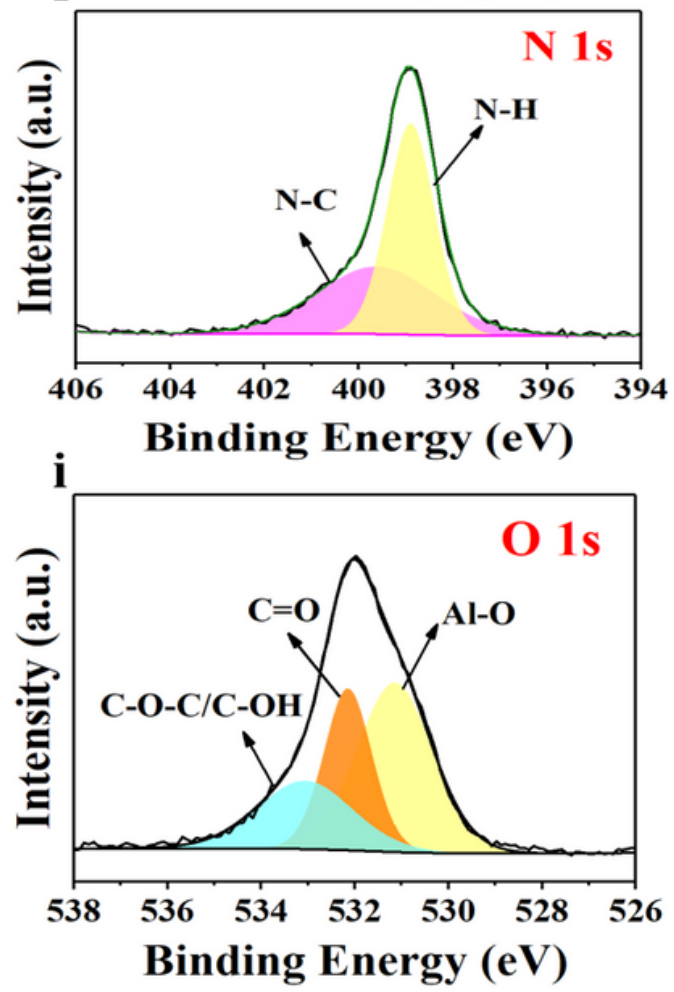

Figure 1

(a) Typical TEM images of M/A-CDs. Scale bar: $20 \mathrm{~nm}$. (b) Particle size distribution, (c) XRD spectra, (d) FTIR spectroscopy, (e) XPS spectra of M/A-CDs. Peak-fitting spectra for 01s (f), C1s (g), N1s (h), Al 2ps (i) of $M / A-C D s$. 

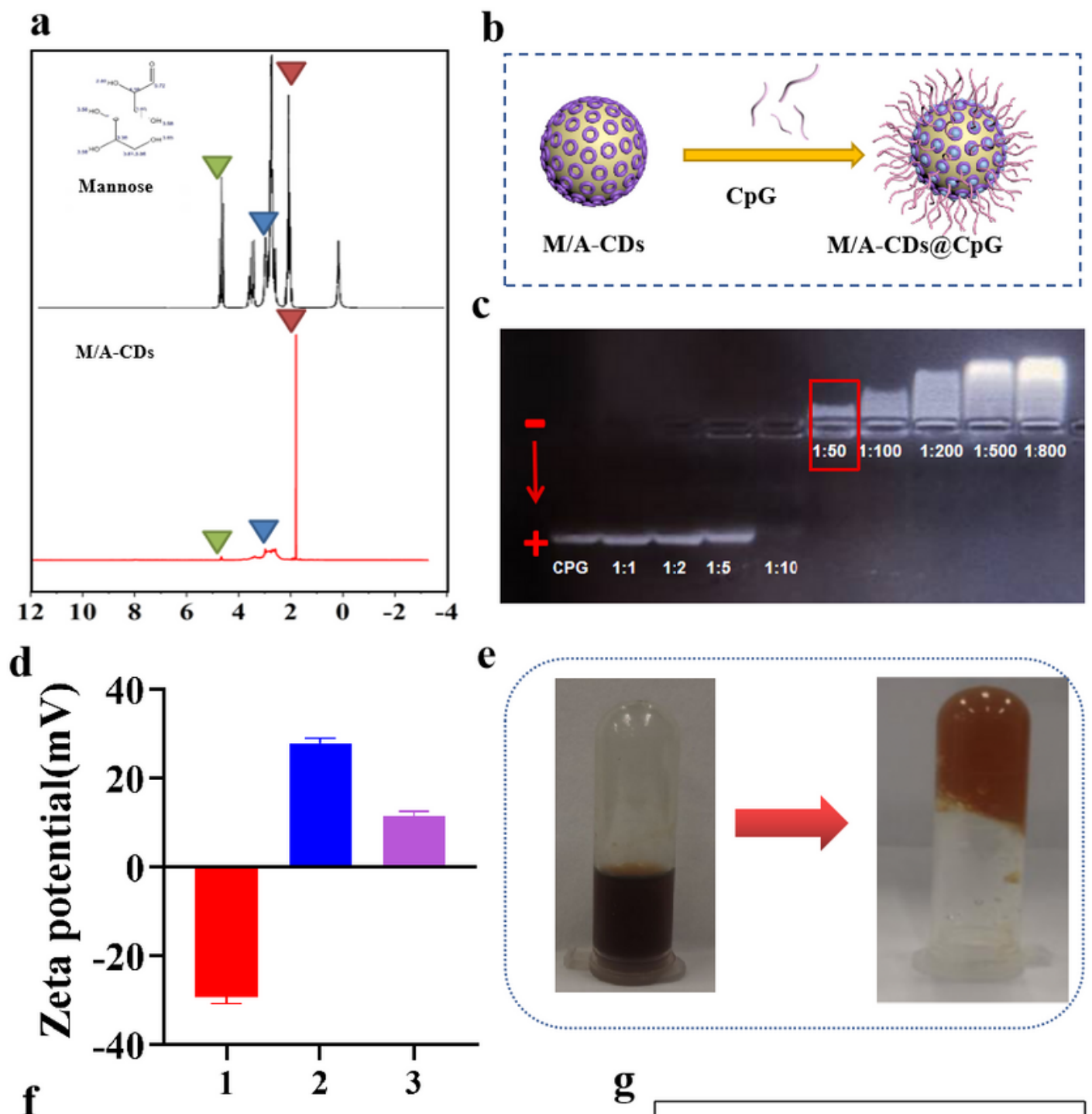

e
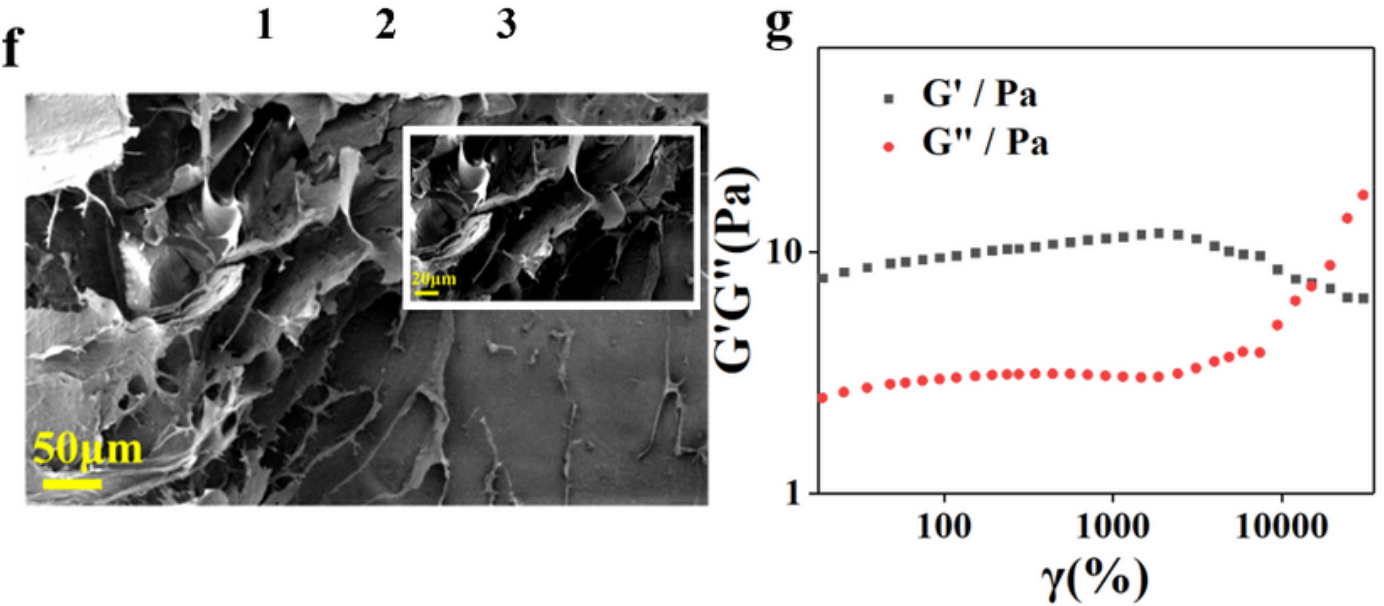

Figure 2

(a) NMR images of M/A-CDs. (b) Schematic diagram of CpG ODNs loaded on M/A-CDs. (c) Gel retardation assay of $M / A-C D$ s and $C p G$ ODNs with various mass ratios. (d) Zeta potential of M/ACDs@CpG (1: CpG, 2: M/A-CDs, 3: M/A-CDs:CpG=100:1). (e) Comparative photos of iCD@Gel before and after gelation. (f) Typical SEM images of iCD@Gel, (g) The strain amplitude sweep test at a fixed angular frequency $(1 \mathrm{~Hz})$ at $37^{\circ} \mathrm{C}$. 

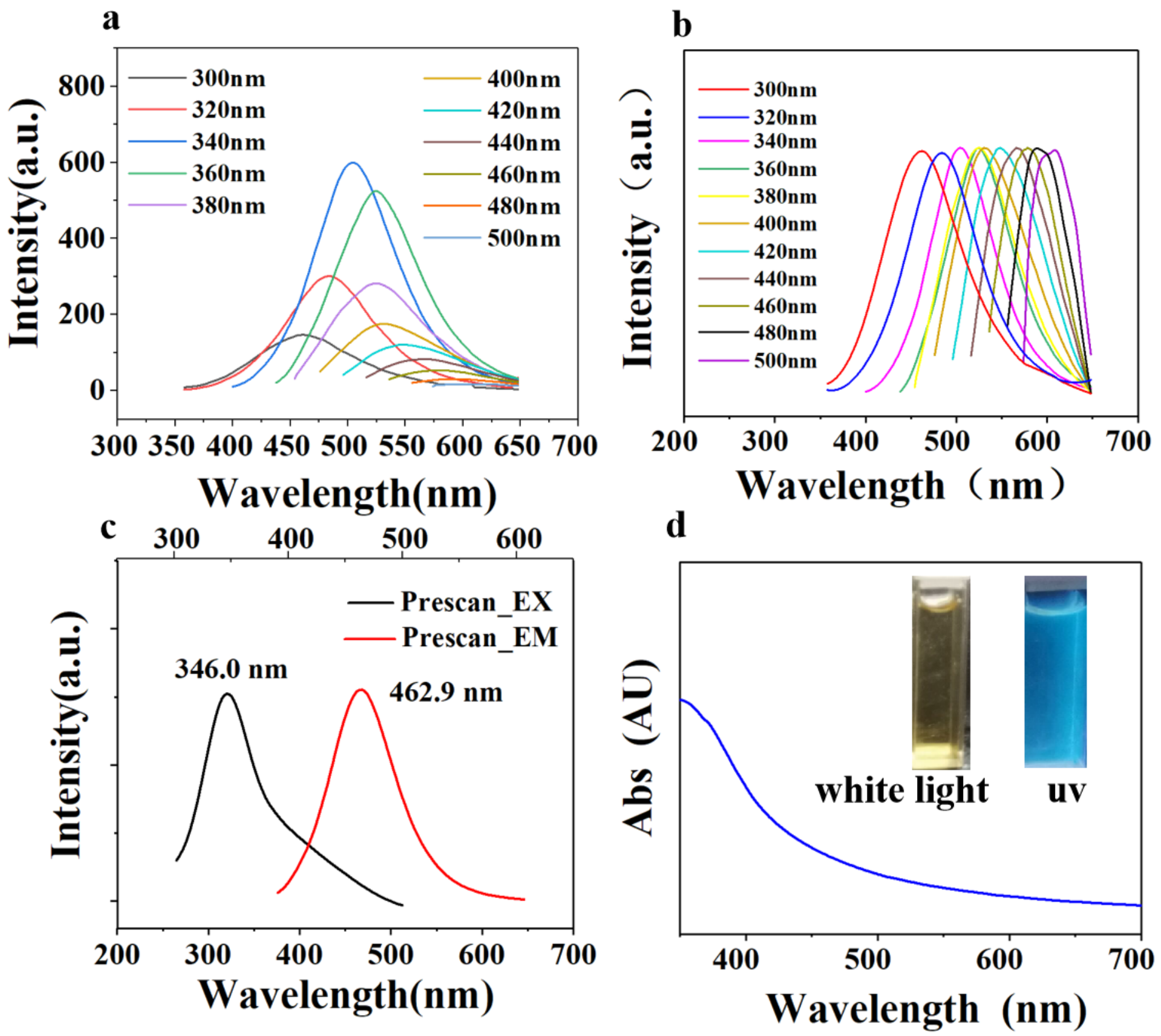

Figure 3

Optical properties of M/A-CDs. (a) Photoluminescence spectra of M/A-CDs at different excitation wavelengths from $300 \mathrm{~nm}$ to $500 \mathrm{~nm}$ (PL). (b) Normalized PL spectra of M/A-CDs. (c) Maximum emission spectra of M/A-CDs at maximum excitation wavelength. (d) UV-visible absorption spectra of M/A-CDs. 

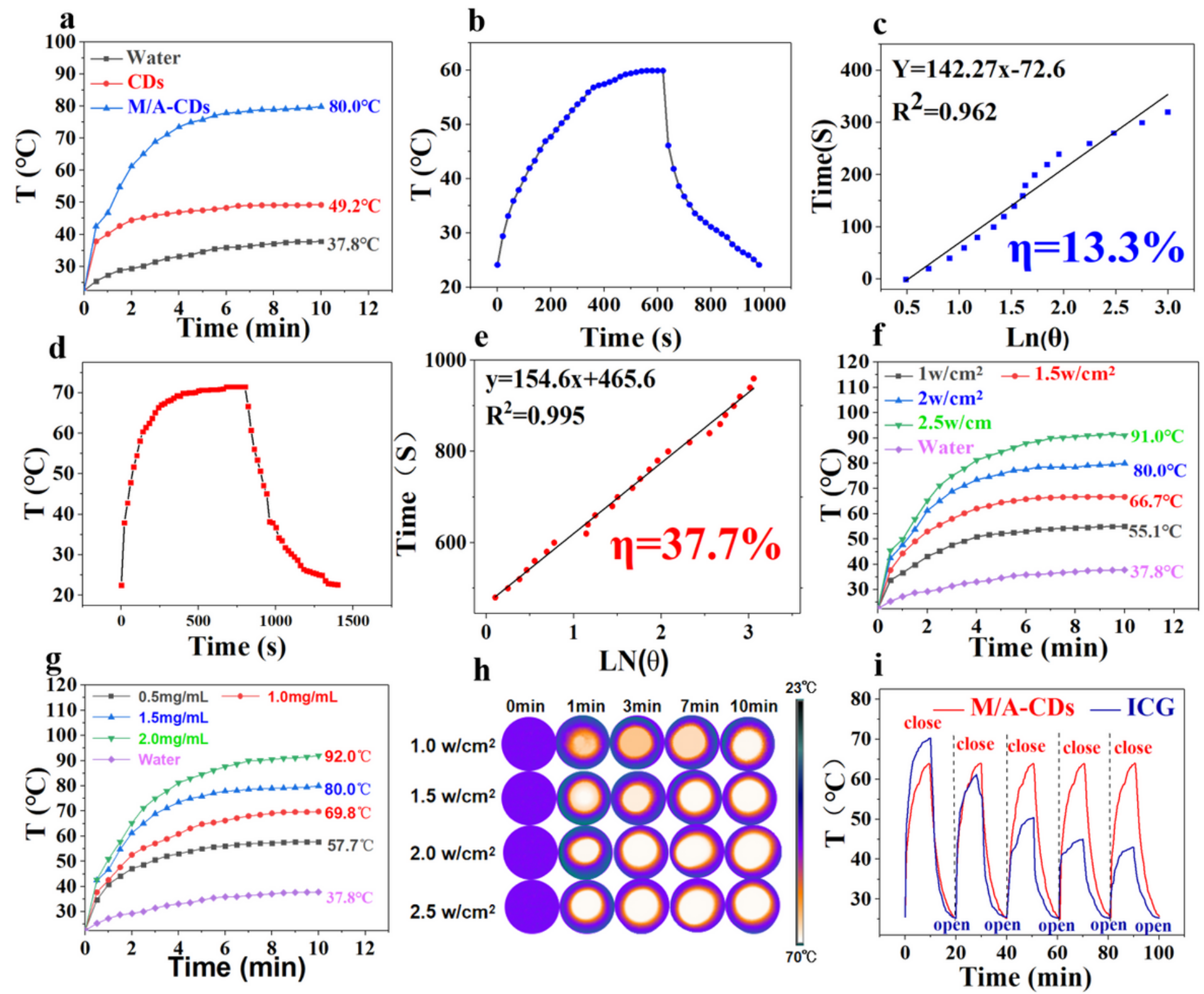

Figure 4

Photothermal performance of M/A-CDs. (a) The temperature curves of the M/A-CDs and the CDs. (b) The temperature curve of CDs solution treated with an NIR laser $\left(808 \mathrm{~nm}, 2 \mathrm{~W} / \mathrm{cm}^{2}\right)$ for $600 \mathrm{~s}$ and left to cool down and (c) linear time data versus - $\ln \theta$ obtained from the cooling stage. (d) The temperature curve of M/A-CDs solution treated with an NIR laser $\left(808 \mathrm{~nm}, 2 \mathrm{~W} / \mathrm{cm}^{2}\right)$ for $600 \mathrm{~s}$ and left to cool down and (e) linear time data versus - $\ln \theta$ obtained from the cooling stage. (f) The temperature curves of M/A-CDs (1.5 $\mathrm{mg} / \mathrm{mL}$ ) solution under NIR irradiation of $808 \mathrm{~nm}$ with different power densities $\left(0-2.5 \mathrm{~W} / \mathrm{cm}^{2}\right)$. (g) The temperature curves of M/A-CDs solution with different concentrations $(0-2 \mathrm{mg} / \mathrm{mL})$ under NIR irradiation of $2 \mathrm{~W} / \mathrm{m}^{2}$ power density. (h) Thermal images of M/A-CDs solution under diferent laser power density. (i) The temperatur curves of M/A-CDs solution after five laser on/off cycles. 
a

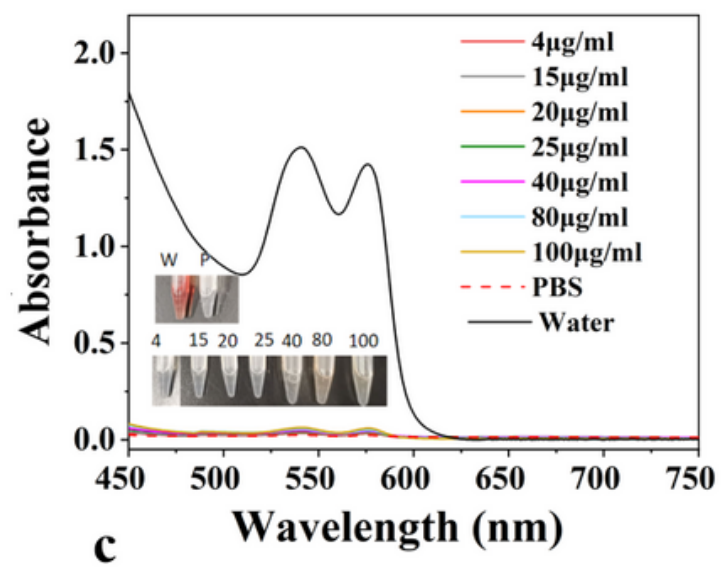

b

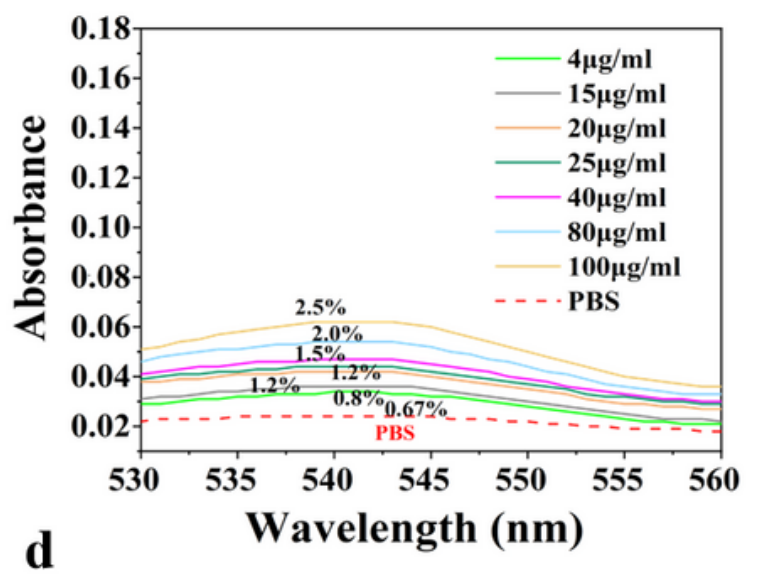

d

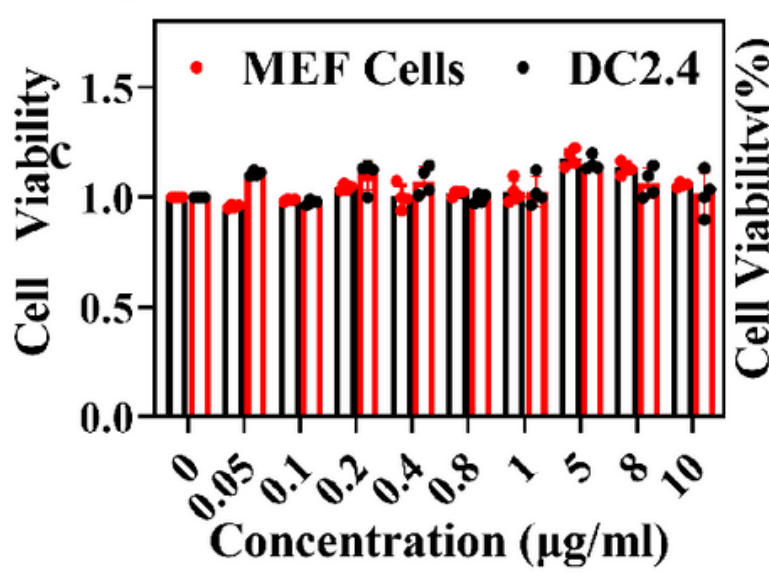

200

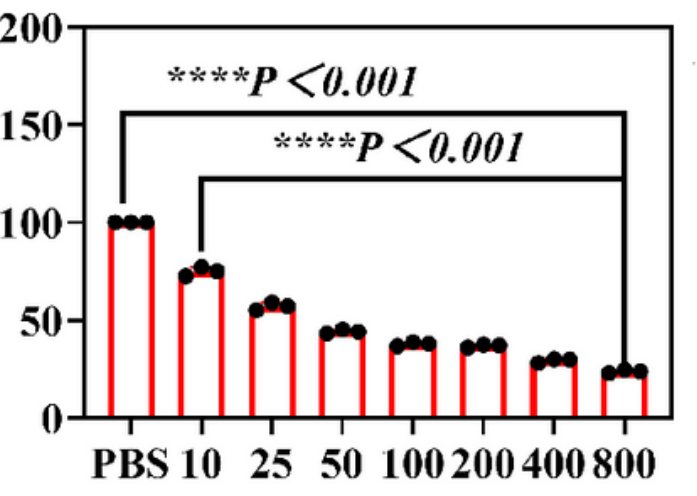

Concentrations $(\mu \mathrm{g} / \mathrm{mL})$
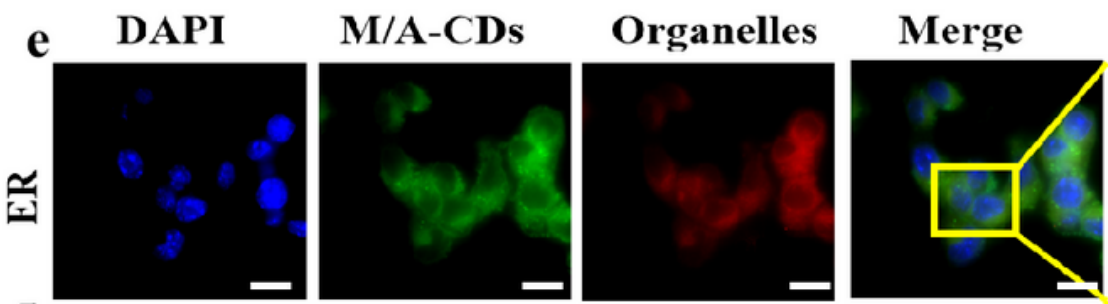

Merge
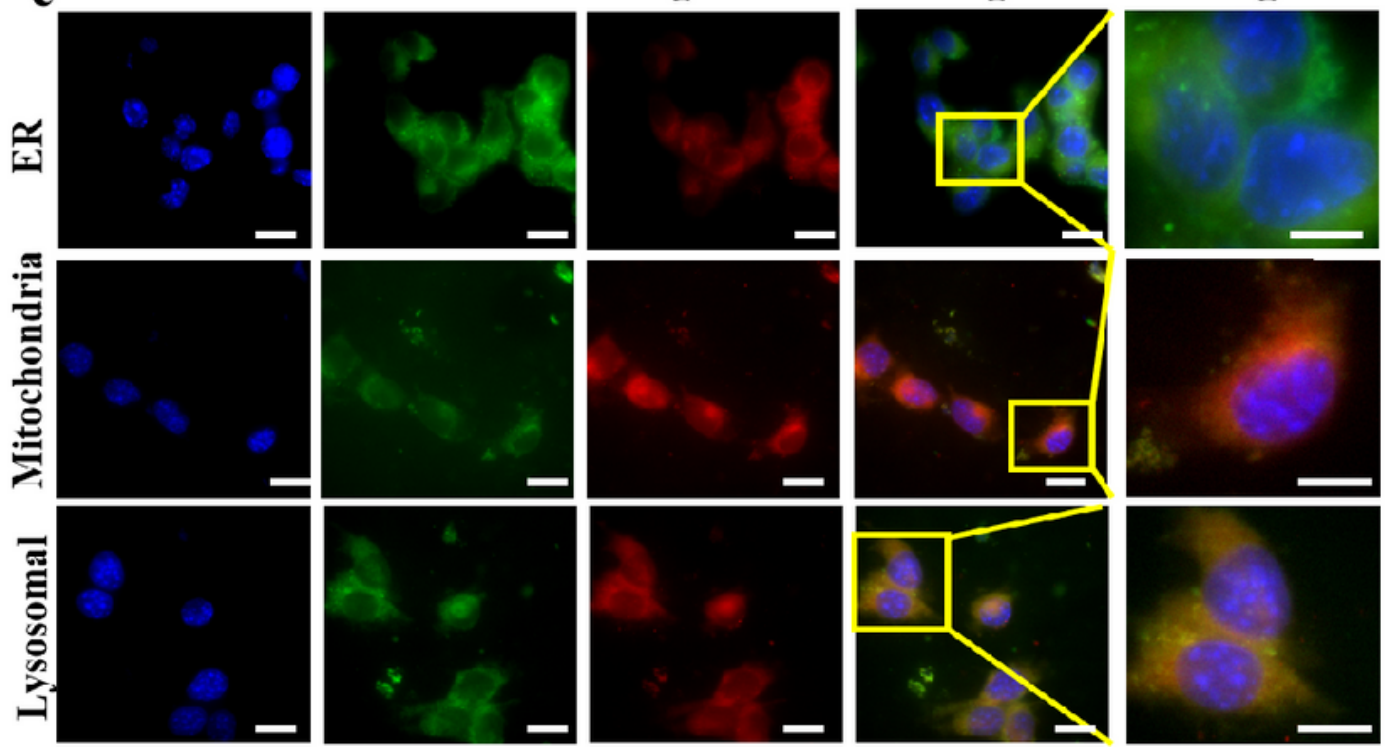

Figure 5

(a) Blood compatibility of M/A-CDs at different concentrations and (b) following amplified version (530$560 \mathrm{~nm}$ ). P, PBS. W, water. (c) Cytocompatibility of M/A-CDs at different concentrations. (d) Effect of $\mathrm{M} / \mathrm{A}-\mathrm{CD}$ s on $4 T 1$ cells under $808 \mathrm{~nm}$ laser irradiation at power of $2 \mathrm{~W} / \mathrm{m}^{2}$. (e) Fluorescence images of $4 \mathrm{~T} 1$ cells co-cultured with M/A-CDs after different organelle probe labeling. 


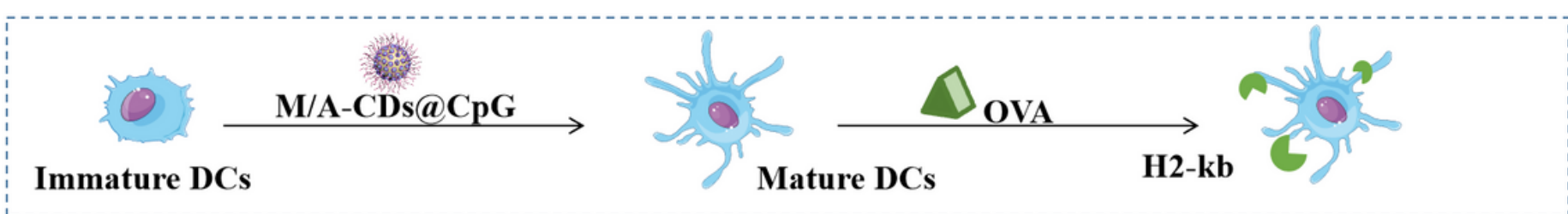

b
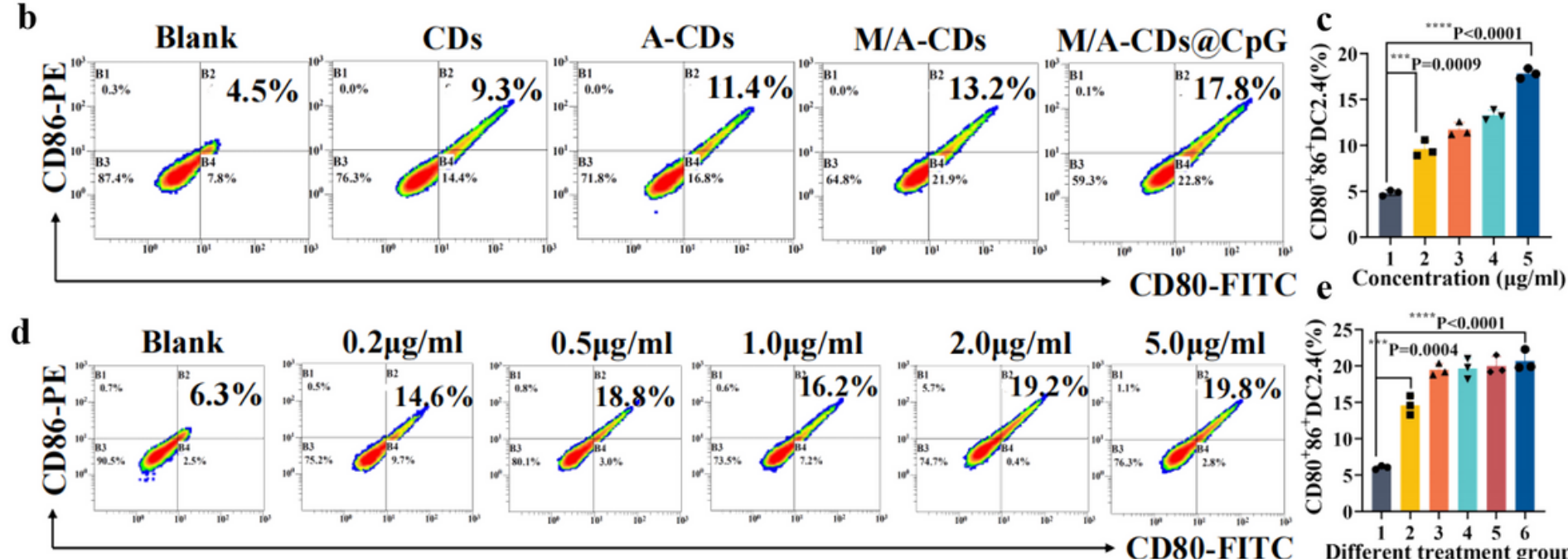

$2.0 \mu \mathrm{g} / \mathrm{ml}$

$5.0 \mu \mathrm{g} / \mathrm{ml}$

f
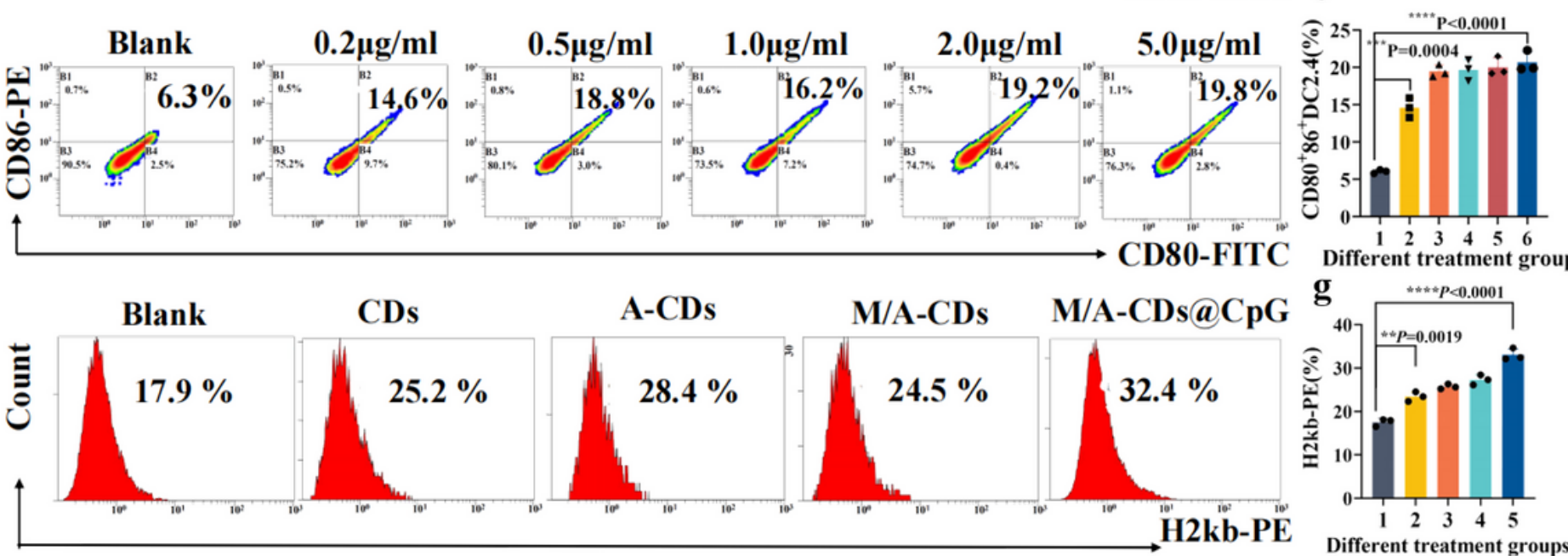

CD80-FITC Different treatment groups

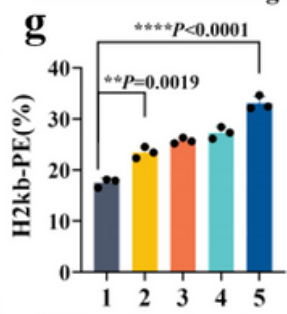

Different treatment groups

Figure 6

The regulation of M/A-CDs@CpG on DC cells maturation. (a) Schematic illustration of the experimental design. Representative flow cytometry plots (b) and the statistic data (c) of the $C D 80^{+} 86^{+}$on the surface of DC2.4 cells after different treatments (1, Blank. 2, CDs. 3, A-CDs. 4, M/A-CDS. 5, M/A-CDs@CpG).

Representative flow cytometry plots (d) and (e) statistic data of $C D 80^{+} 86^{+}$on the surface of DC2.4 cells after co-cultured with different concentrations of M/A-CDs@CpG (1, Blank. 2, $0.2 \mu \mathrm{g} / \mathrm{ml} .3,0.5 \mu \mathrm{g} / \mathrm{ml}$. 4, $1.0 \mu \mathrm{g} / \mathrm{ml}$. 5, $5.0 \mu \mathrm{g} / \mathrm{ml}$ ) . (f) Flow pattern and (g) Quantitative analysis of H2kb antigen expression on the surface of DC2.4 cells after different treatmentsb(1: Blank, 2: CDs, 3: A-CDs, 4: M/A-CDS, 5:M/ACDs@CpG ). Data are presented as the mean \pm s.d. Statistical significances were calculated by one-way analysis of variance with Tukey's post hoc test and two-tailed Student's t-test. ${ }^{*} \mathrm{P}<0.05$, ${ }^{\star *} \mathrm{P}<0.01$, ${ }^{\star \star *} \mathrm{P}<$ $0.001, \star \star \star \star P<0.0001$. 


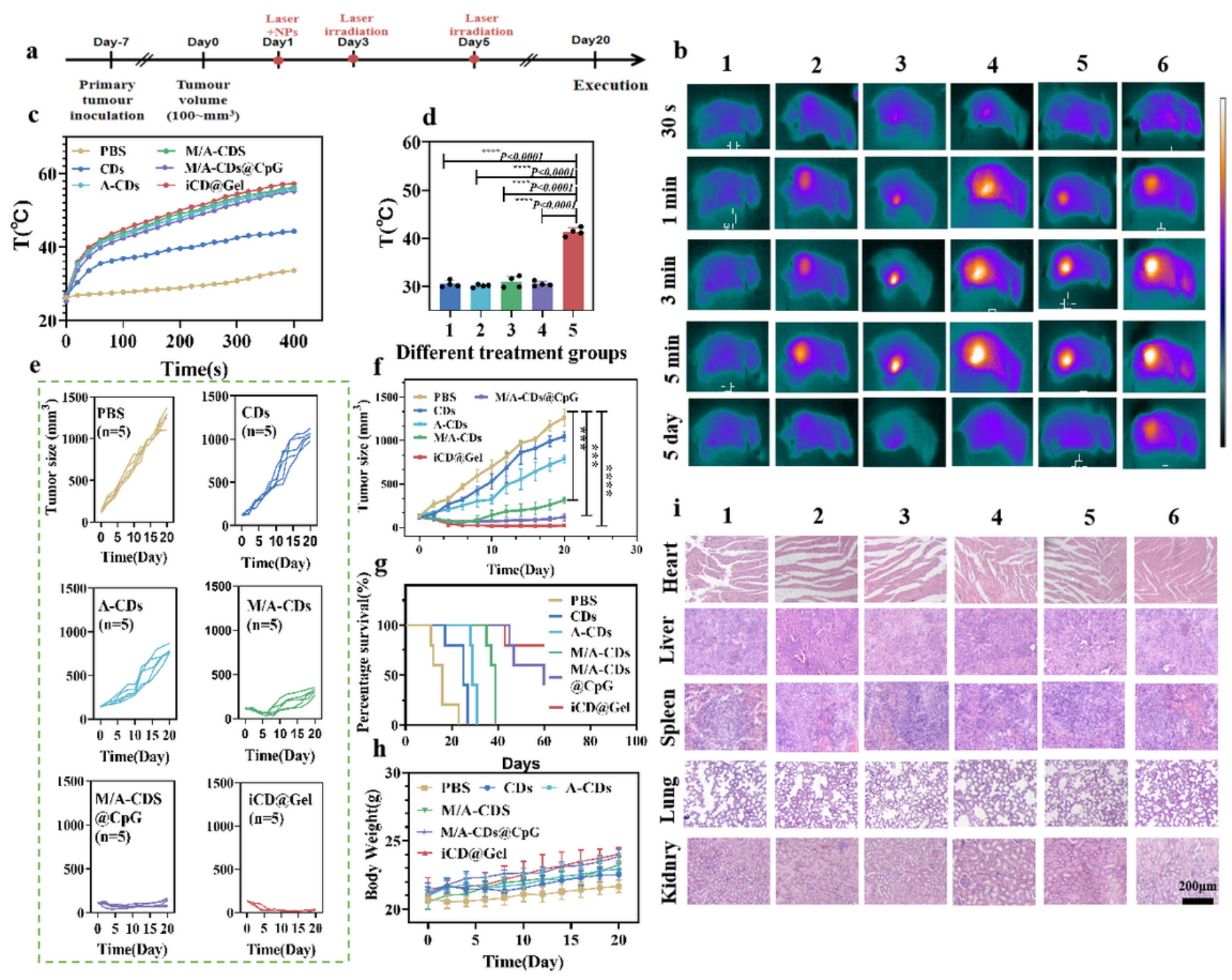

Figure 7

In vivo photothermal effect of iCD@Gel and its inhibitory effect on tumor growth. (a) Schematic illustration and experimental design. (b) Thermal images of mice after in situ injectin of iCD@Gel under $808 \mathrm{~nm}$ laser irradiation with a power density of $1.5 \mathrm{~W} / \mathrm{cm}^{2}$ at different time points (1, PBS. 2, CDs. 3, ACDs. 4, M/A-CDs. 5, M/A-CDs@CpG.6, iCD@Gel). (c) Temperature curves of different treatment groups at different time points under $808 \mathrm{~nm}$ near-infrared laser irradiation $(1 \mathrm{mg} / \mathrm{kg})$. (d) Temperature changes of different treatment groups after 5 days under $808 \mathrm{~nm}$ near-infrared laser irradiation. (e) Tumor volume curves of each mouse within 20 days of treatment. (f) Average tumor volume curves each mouse within 20 days of treatment. $(n=5)$. (g) Body-weight change of mice in different groups. (h) Survival curves of mice in the different groups. (h) H\&E-stained images of the major organs in the different treatment

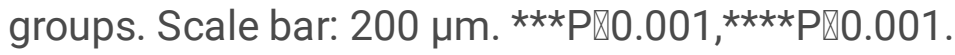


$\mathbf{a}$

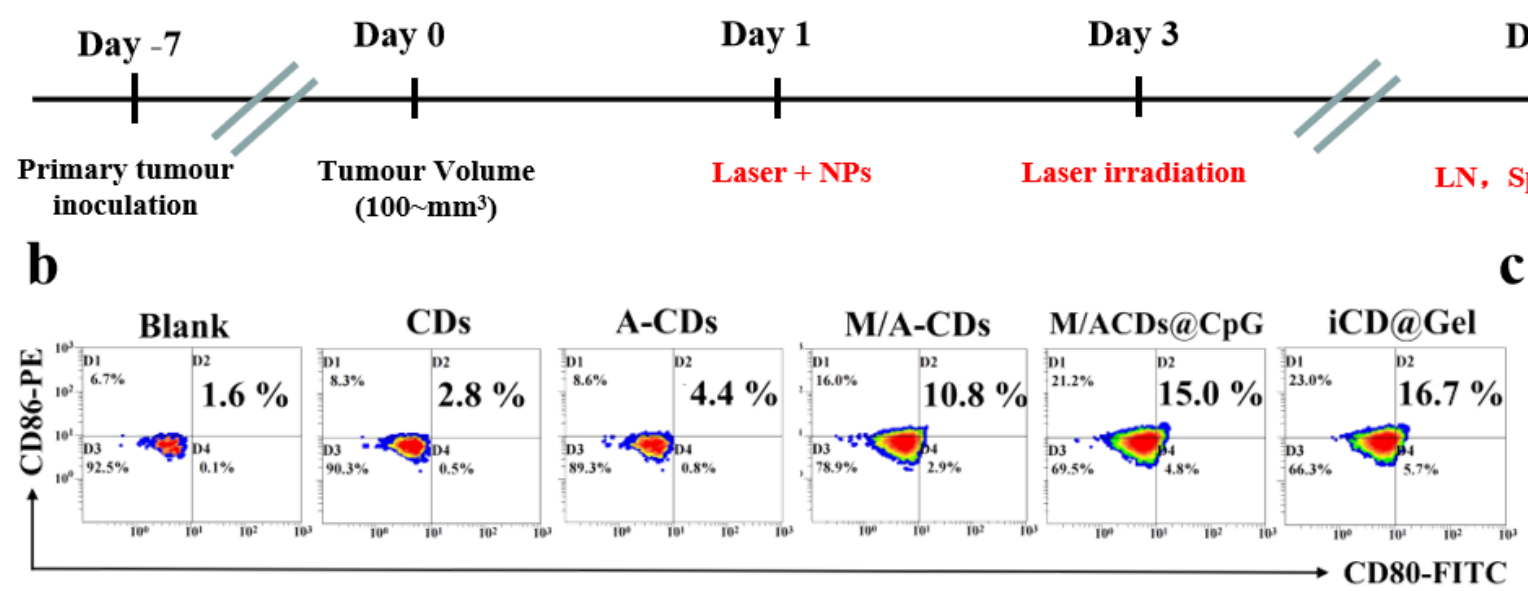

Day 7
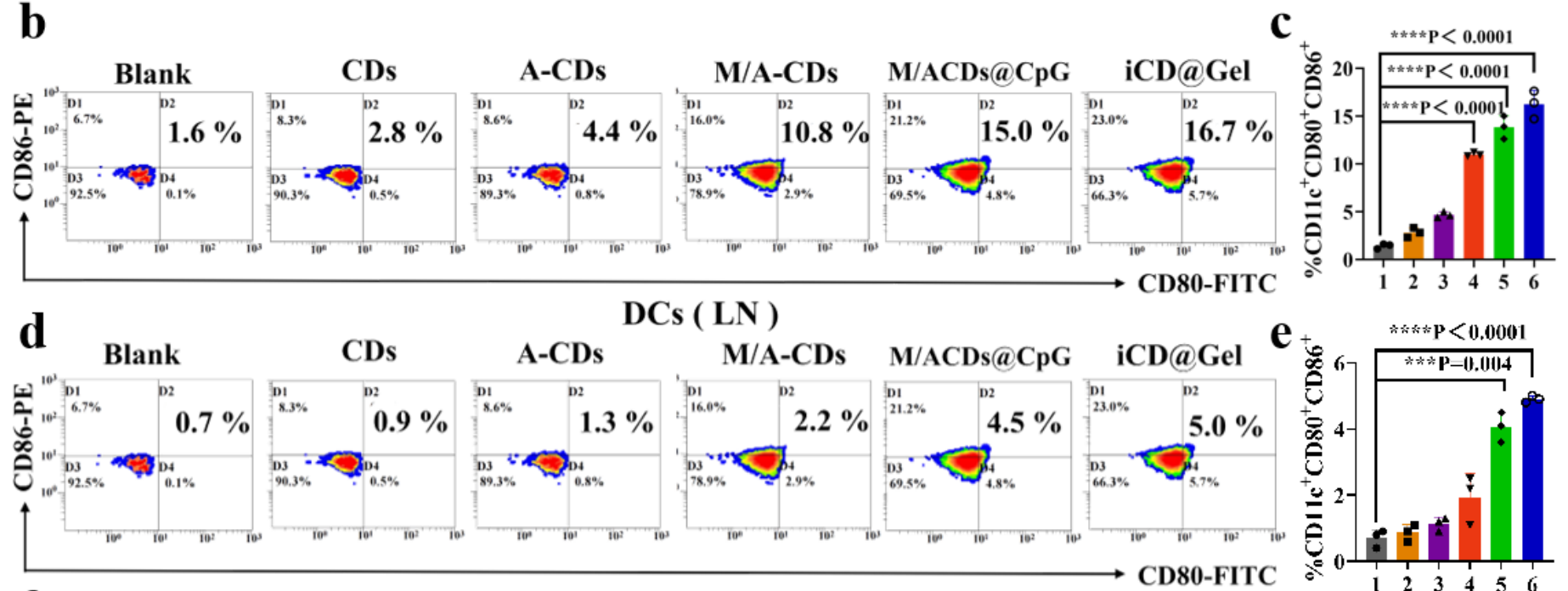

$\mathbf{f}$
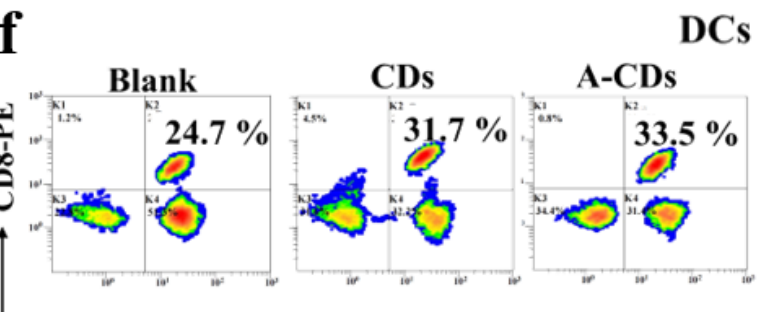

Spleens )

$\mathrm{CD8}^{+} \mathrm{T}$ Cells ( LN )

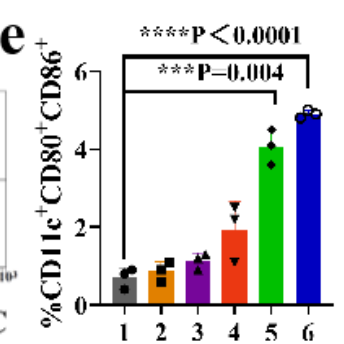

h
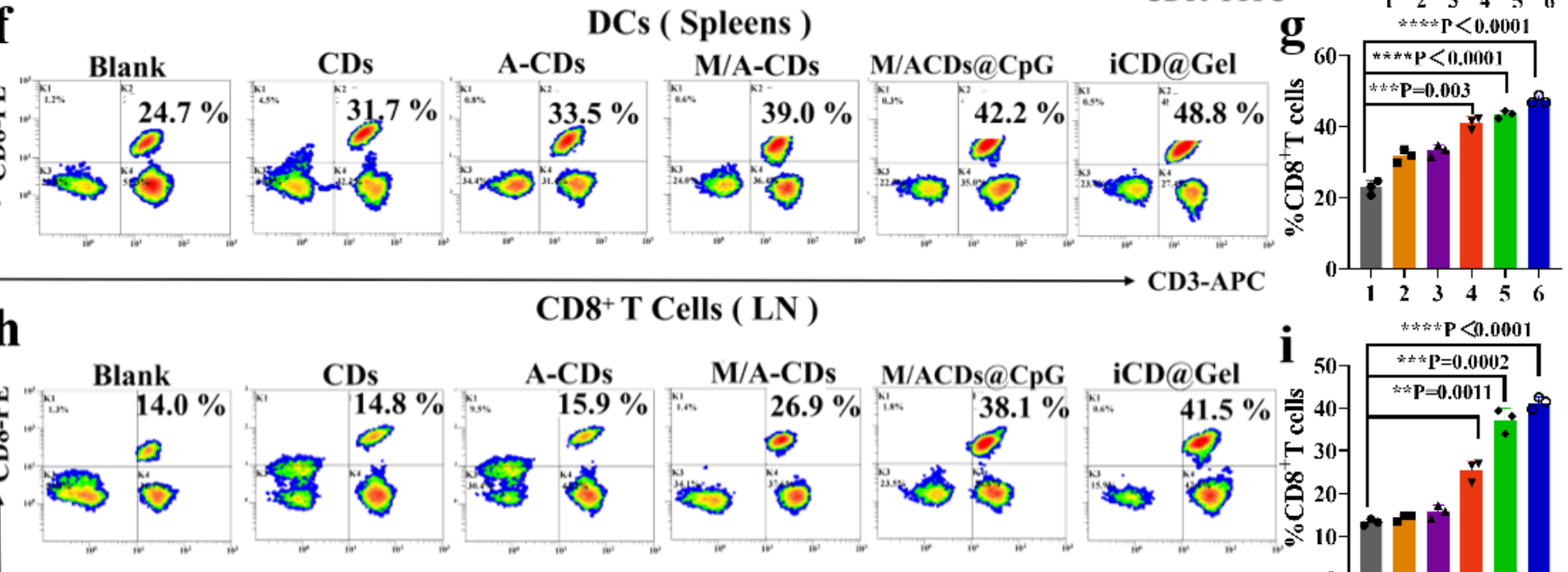

CD8+T Cells( Spleens )

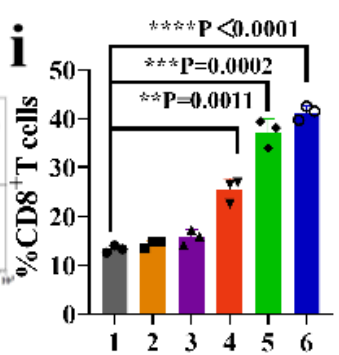

\section{Figure 8}

The immunoregulatory effect of iCD@Gel in vivo. (a) Experimental design. (b) The representative flow cytometry and (c) quantitative analysis of $\mathrm{CD} 80^{+} \mathrm{CD} 86^{+}$cells from the lymph node. (d) The representative flow cytometry and (e) quantitative analysis of $\mathrm{CD} 80^{+} \mathrm{CD} 86^{+}$cells from the spleens. (f) The representative flow cytometry and $(\mathbf{g})$ quantitative analysis of $\mathrm{CD}^{+} \mathrm{CD} 8^{+} \mathrm{T}$ cells from the lymph node. (h) The representative flow cytometry and (i) quantitative analysis of $C D 3^{+} C D 8^{+} T$ cells from spleens.(Groups: 1 , Blank. 2, CDs. 3, A-CDs. 4, M/A-CDs. 5, M/A-CDs@CpG.6, iCD@Gel.)

\section{Supplementary Files}


This is a list of supplementary files associated with this preprint. Click to download.

- Scheme1.png 